\title{
Secretoneurin promotes neuroprotection and neuronal plasticity via the Jak2/Stat3 pathway in murine models of stroke
}

\author{
Woei-Cherng Shyu, ${ }^{1}$ Shinn-Zong Lin,, ${ }^{1}$ Ming-Fu Chiang, ${ }^{2}$ Der-Cherng Chen, ${ }^{3}$ Ching-Yuan Su, ${ }^{4}$ \\ Hsiao-Jung Wang, ${ }^{1}$ Ren-Shyan Liu, ${ }^{5}$ Chang-Hai Tsai, ${ }^{6}$ and Hung Li ${ }^{4}$ \\ ${ }^{1}$ Center for Neuropsychiatry, China Medical University and Hospital, Taichung, Republic of China. ${ }^{2}$ Department of Neurosurgery, \\ Mackay Memorial Hospital, Mackay Junior College of Nursing, Taipei, Republic of China. ${ }^{3}$ Department of Neurosurgery, \\ Buddhist Tzu-Chi General Hospital, Tzu-Chi University, Hualien, Republic of China. ${ }^{4}$ Institute of Molecular Biology, Academia Sinica, Taipei, \\ Republic of China. ${ }^{5}$ Department of Nuclear Medicine, Taipei Veterans General Hospital, National Yang-Ming University, Taipei, Republic of China. \\ ${ }^{6}$ Department of Pediatrics, China Medical University and Hospital, Taichung, Republic of China.
}

\begin{abstract}
Secretoneurin (SN), a neuropeptide derived from secretogranin II, promotes neurite outgrowth of immature cerebellar granule cells. SN also aids in the growth and repair of neuronal tissue, although the precise mechanisms underlying the promotion of brain tissue neuroprotection and plasticity by SN are not understood. Here, in a rat model of stroke and in ischemic human brain tissue, $\mathrm{SN}$ was markedly upregulated in both neurons and endothelial cells. SN-mediated neuroprotection rescued primary cortical cell cultures from oxygen/ glucose deprivation. SN also induced expression of the antiapoptotic proteins Bcl-2 and Bcl-xL through the Jak2/Stat3 pathway and inhibited apoptosis by blocking caspase-3 activation. In addition, rats with occluded right middle cerebral arteries showed less cerebral infarction, improved motor performance, and increased brain metabolic activity following i.v. administration of SN. Furthermore, SN injection enhanced stem cell targeting to the injured brain in mice and promoted the formation of new blood vessels to increase local cortical blood flow in the ischemic hemisphere. Both in vitro and in vivo, SN not only promoted neuroprotection, but also enhanced neurogenesis and angiogenesis. Our results demonstrate that $\mathrm{SN}$ acts directly on neurons after hypoxia and ischemic insult to further their survival by activating the Jak2/Stat3 pathway.
\end{abstract}

\section{Introduction}

Secretoneurin (SN) is a 33-amino acid neuropeptide produced by endoproteolytic processing of chromogranin/secretogranin family proteins, which are found in large dense-core vesicles in a wide variety of cell types of the endocrine tissue and nervous system $(1,2)$. In several recent reports, chromogranin/SN proteins have been found to be closely correlated with synaptic disturbance caused by neuronal/ glial and inflammatory mechanisms in conditions such as Alzheimer disease (3-5). It has also been reported that $\mathrm{SN}$ can promote the neurite outgrowth of immature cerebellar granule cells (6). Furthermore, in a recent report, increased expression of $\mathrm{SN}$ was found in an animal model after transient forebrain ischemia (7). Although there is much evidence suggesting an important role for SN in the physiology and pathophysiology of the nervous system, its precise role in neuroprotection and neuronal plasticity has not been clarified.

Although several articles have reported a correlation between SN and neurological diseases including Alzheimer disease (3-5), Parkinson disease (8), and epilepsy $(9,10)$, little literature has examined the role of SN in stroke (7). Human stroke is a leading cause of death and disability worldwide (11), and as yet there is no effective

Nonstandard abbreviations used: BMSC, bone marrow stem cell; $\mathrm{CBF}$, cerebral blood flow; CCA, common carotid artery; ${ }^{18} \mathrm{FDG},\left[{ }^{18} \mathrm{~F}\right]$ fluoro-2-deoxyglucose; GFAP, glial fibrillary acidic protein; IHC, immunohistochemical, immunohistochemistry; INPC, intrinsic neural progenitor cell; LDH, lactate dehydrogenase; MAP-2, microtubular-associated protein-2; MCA, middle cerebral artery; Neu-N, neuronal nucleus; OGD, oxygen/glucose deprivation; p-, phosphorylated; PCC, primary cortical cell; SN, secretoneurin; SN-IR, SN-immunoreactive, SN-immunoreactivity.

Conflict of interest: The authors have declared that no conflict of interest exists. Citation for this article: J. Clin. Invest. 118:133-148 (2008). doi:10.1172/JCI32723. treatment that enhances stroke recovery. One potential strategy for the treatment of stroke is transplantation of bone marrow stem cells (BMSCs) (12) leading to enhancement of neurogenesis and angiogenesis, which have been demonstrated to promote plasticity and assist in the recovery from stroke $(13,14)$. Recently, the role of bone marrow-derived circulating progenitor cells in postnatal angiogenesis and neurogenesis has been clearly demonstrated in hind-limb, myocardial, and cerebral ischemia (15-17). Due to the effects of SN on the induction of vasculogenesis through activation of the Akt signaling pathway (18), the mobilization of bone marrow-derived endothelial progenitor cells (19), and the increased SN expression seen in ischemic tissue (7), we hypothesized that SN might enhance neuroprotection and plasticity in the cerebral ischemic animal model. Furthermore, some growth factors may enhance the bone marrow-derived progenitor cells' proliferation and angiogenesis via activation of the Jak2/Stat3 pathway $(20,21)$. Therefore, in the present study, we have examined the neuroprotective effects of SN against oxygen/glucose deprivation-induced (OGD-induced) neurotoxicity in primary cortical neurons and also analyzed the results of i.v. administration of $\mathrm{SN}$ on cerebral ischemic animals by measuring changes in the extent of induced cerebral infarction and neurological dysfunction. In addition, we also focused on the Jak2/Stat3 pathway to discern the possible molecular mechanism for the neuroprotective role of SN.

\section{Results}

Cerebral ischemia increases the immunoreactivity of SN in buman and rat brains. In order to determine whether cerebral ischemia increases the expression of SN, levels of SN were measured by analysis of 
A
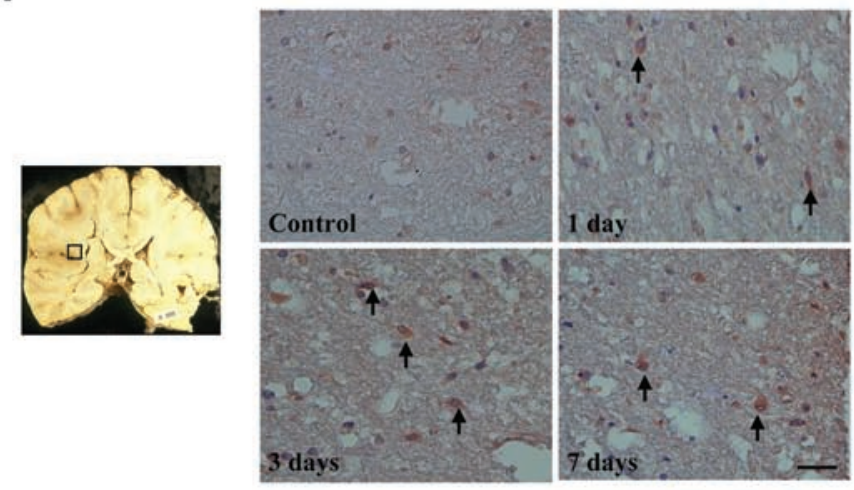

C

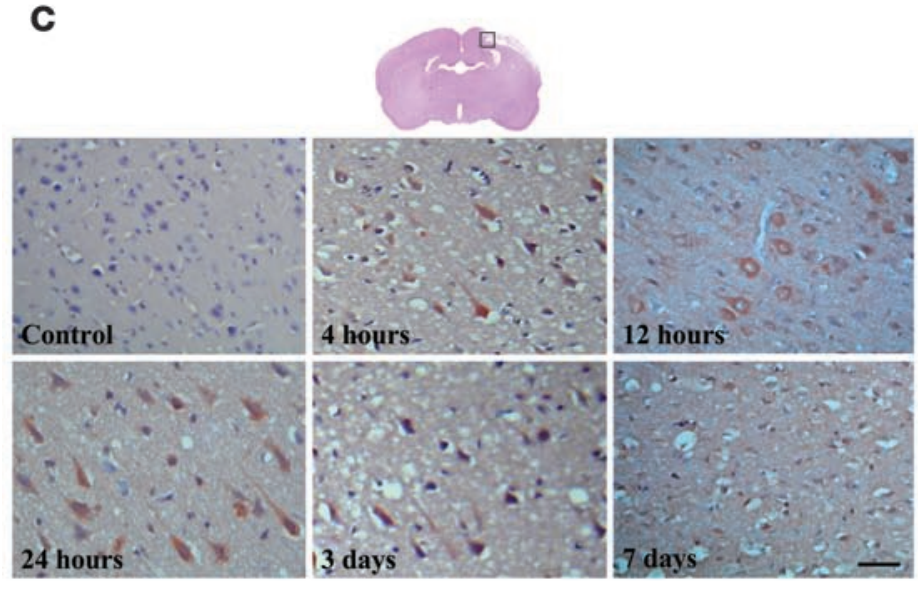

E

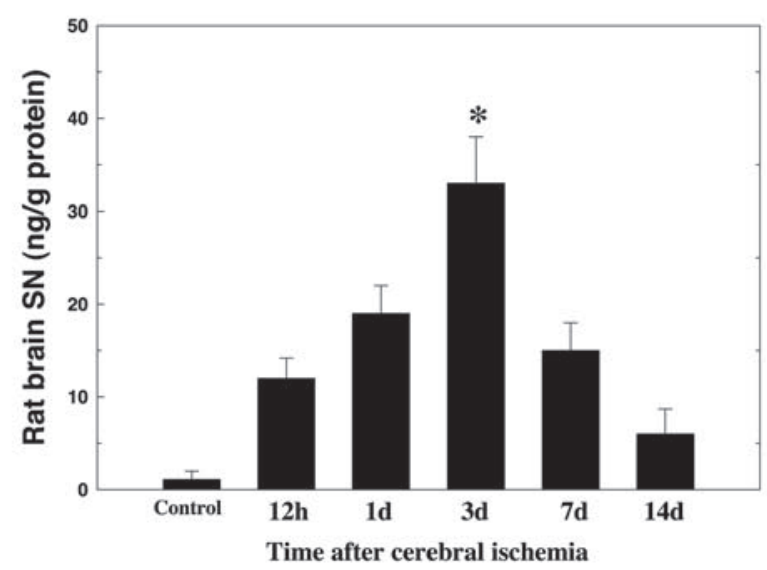

G
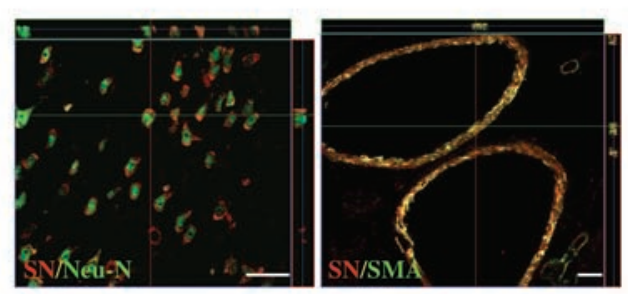

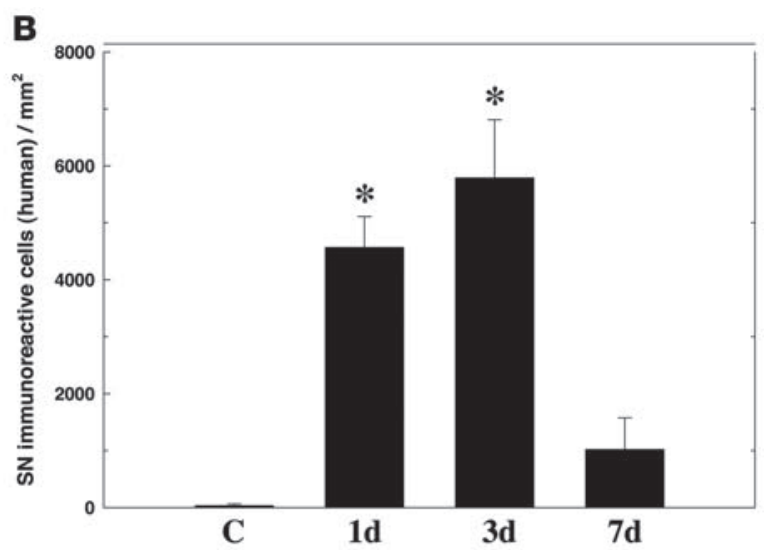

Time after cerebral ischemia
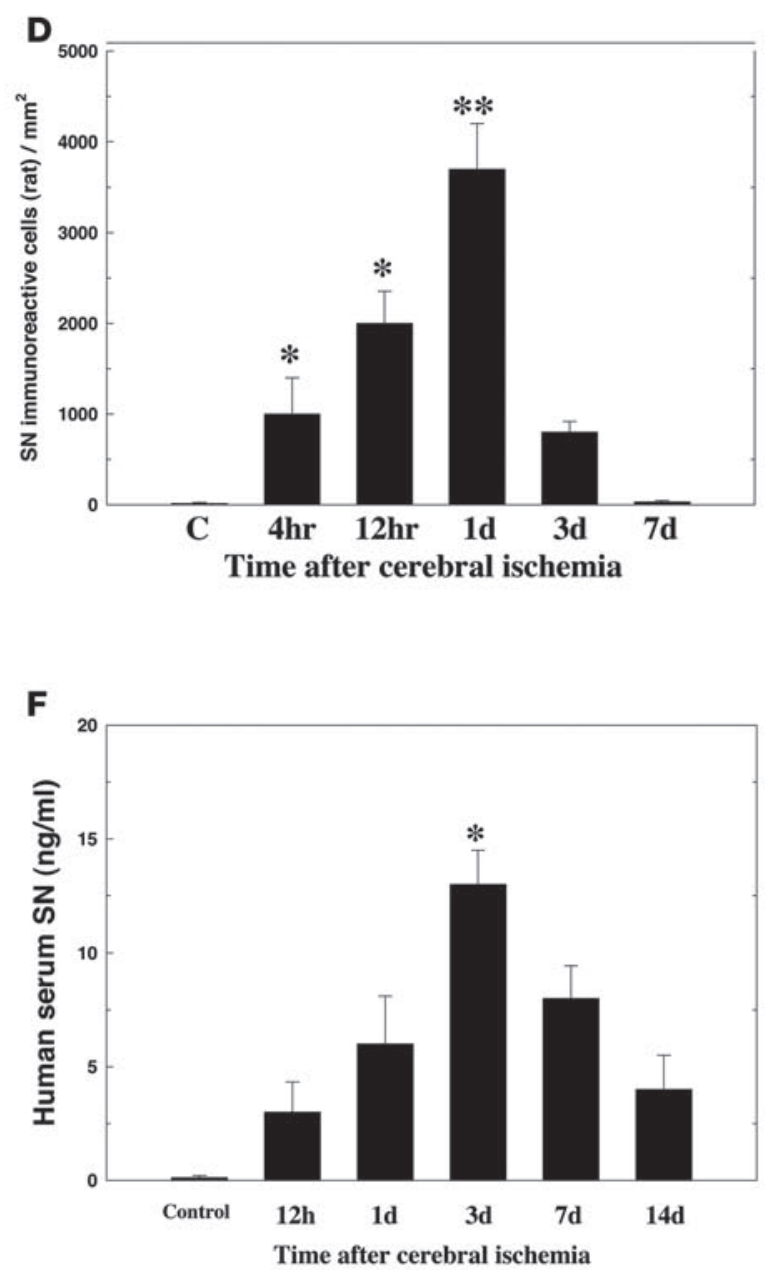


\section{Figure 1}

Cerebral ischemia increases expression of SN in human and rat brain. (A) Representative brain of a stroke patient; boxed region shows infarct area. IHC studies of the penumbral area showed markedly increased SN-IR. (B) SN-IR in the stroke patients' brains significantly increased at 1 and 3 days after cerebral infarction compared with that of controls. (C and D) IHC and quantitative analysis of SN-IR of animal cerebral ischemia (boxed region) showed greater numbers of SN-IR cells compared with controls. (E and F) Measurement of SN level using ELISA at the indicated time points showed a significant increase of SN expression in both ischemic rats' brains and stroke patients' sera compared with controls. (G) Double immunofluorescence with laser-scanning confocal microscopy of ischemic rat brain specimens. The ischemic cortical areas of the rats revealed SN-IR cells coexpressing Neu-N+. Some SN-IR cells showing vascular phenotypes (SMA+ cells) were also found around the perivascular and endothelial regions of the ischemic hemispheres. Data are mean \pm SEM. ${ }^{*} P<0.05,{ }^{\star \star} P<0.01$ vs. control. Scale bars: $50 \mu \mathrm{m}$.

SN-immunoreactivity (SN-IR). Brain samples from human stroke patients at 1,3 , and 7 days after ictus $(n=9)$ and experimental rats at 4 and 12 hours and 1,3 , and 7 days after undergoing cerebral ischemia ( $n=20$; 4 per time point) were examined for SN immunostaining. Significantly more SN-IR cells were found in the penumbral and hippocampal regions of the ischemic brain sample from human stroke patients 1 and 3 days after cerebral infarction than in those of controls ( $n=3$; Figure 1, A and B). In the animal model, greater numbers of SN-IR cells were detected 4 hours $(n=4)$ after cerebral ischemia than in the nonischemic control $(n=6)$. SN-IR cells were mainly detected in the ipsilateral cortex near the infarcted boundary region of the ischemic rat brains (Figure 1, C and D). In addition, there was a time-dependent increase in the number of SN-IR cells found around the penumbral region in ischemic rats; in comparison, nonischemic rats showed no increase (Figure 1D).

Cerebral ischemia enhances expression of $S N$ in the ischemic brain and serum. In order to determine whether the expression of SN was increased in rats and humans, SN levels in brain samples from rats that had undergone cerebral ischemia $(n=20)$ and sera from human stroke patients $(n=20)$ were measured using ELISA. Samples of ischemic rat brains were taken from the cortical region and striatum. Brain samples from homologous areas of rats without middle cerebral artery (MCA) ligation were used as normal controls $(n=6)$. ELISA analysis of SN levels showed that cerebral ischemia in rat brains caused an increase in the expression of $\mathrm{SN}$ in a time-dependent manner (control, $1.1 \pm 0.1 \mathrm{ng} / \mathrm{g}$ protein; 12 hours, $12.1 \pm 2.2 \mathrm{ng} / \mathrm{g}$ protein; 1 day, $19.3 \pm 3.2 \mathrm{ng} / \mathrm{g}$ protein; 3 days, $33.1 \pm 5.2 \mathrm{ng} / \mathrm{g}$ protein; 7 days, $15.4 \pm 3.3 \mathrm{ng} / \mathrm{g}$ protein; 14 days, $6.2 \pm 2.7 \mathrm{ng} / \mathrm{g}$ protein; Figure 1E), which was in agreement with our immunohistochemical (IHC) studies of an ischemic rat's brain that showed an increase in SN-IR (Figure 1A). In addition, sera from human stroke patients were also collected at different time points. Control serum samples were obtained from 6 healthy age-matched subjects. The level of SN in human stroke patients' sera (12 hours, $3.1 \pm 1.3 \mathrm{ng} / \mathrm{ml} ; 1$ day, $6.4 \pm 2.1 \mathrm{ng} / \mathrm{ml} ; 3$ days, $13.3 \pm 1.5 \mathrm{ng} / \mathrm{ml} ; 7$ days, $8.5 \pm 1.4 \mathrm{ng} / \mathrm{ml} ; 14$ days, $4.1 \pm 1.5 \mathrm{ng} / \mathrm{ml})$ was significantly higher than that of the controls $(0.12 \pm 0.09 \mathrm{ng} / \mathrm{ml}$; Figure $1 \mathrm{~F}$ ) and peaked at 3 days after stroke onset.

Immunoreactivity of $S N$ colocalizes to neurons and endothelial cells after cerebral ischemia. In order to identify which cerebral cells expressed SN after cerebral ischemia, double immunofluorescence was performed on brain specimens of ischemic rats with laser-scanning confocal microscopy. Ischemic cortical areas of the rats revealed many SN-IR cells coexpressing a neuronal nucleus-positive $\left(\mathrm{Neu}-\mathrm{N}^{+}\right)$neuronal phenotype (163 \pm 26 cells $/ \mathrm{mm}^{2}$; Figure $\left.1 \mathrm{G}\right)$. Some SN-IR cells showing $\mathrm{SMA}^{+}$vascular phenotypes were also found around the perivascular and endothelial regions (Figure 1G) of the ischemic hemispheres.

Neuroprotective effect of SN in primary cortical cell cultures. To evaluate the neuroprotective effect of SN in vitro, lactate dehydrogenase (LDH) activity and neuronal survival (positive microtubular-associ- ated protein-2 [MAP-2] immunoreactivity) under OGD-induced neurotoxic conditions were measured in primary cortical cells (PCCs) with or without SN treatment (Figure 2, A-C). Treatment with $1 \mu \mathrm{g} / 1$ $\mathrm{SN}$ at 20 minutes prior to OGD significantly reduced LDH activity in cultures compared with the control group (Figure 2D).

To analyze the effect of SN on neuronal survival after OGD, the number of MAP-2-immunoreactive cells was analyzed quantitatively. Pretreatment with $1 \mu \mathrm{g} / \mathrm{l} \mathrm{SN}$ at 20 minutes prior to OGD significantly increased MAP-2-immunoreactive cell density in PCCs compared with the control group (Figure 2E). However, treatment with SN after OGD did not show a significantly neuroprotective effect in PCCs.

Neuroprotection by SN might be through an antiapoptotic effect in PCCs. In order to examine whether the neuroprotective effect of $\mathrm{SN}$ is caused by blocking an apoptotic pathway, an immunofluorescent study of caspase- 3 under OGD-induced neurotoxic conditions was performed in PCCs with or without SN treatment. In the OGD environment, PCCs treated with SN for 12 hours showed significantly reduced caspase- 3 activity using a fluorimetric method compared with the control group (Figure $2 \mathrm{~F}$ ). There was a significant reduction of caspase- $3^{+}$immunofluorescent cells in the SN-treated group compared with the control group (Figure $2 \mathrm{G}$ ).

In addition, to investigate the molecular mechanism by which $\mathrm{SN}$ exerts an antiapoptotic effect on PCCs, we studied the expression of apoptosis-related proteins by Western blot analysis. Expression levels of antiapoptotic proteins such as Bcl-2 were lower when PCCs were subjected to OGD; this reduction was considerably inhibited by pretreatment with SN for 12 hours (Figure 2, H and I).

SN activates the Jak2/Stat3 signal transductional pathway in PCCs. In order to examine whether $\mathrm{SN}$ exerts neuroprotective effects through specific cellular signals, the expression of activated protein kinases of both Jak2 and Stat3 in PCCs were measured after total protein was extracted. We found that the level of activated Jak2 and Stat 3 after SN treatment increased in a time- and dosedependent manner (Figure 2, J-M). The expression of Stat3 and Jak2 was not significantly different between SN-treated and control cells (Figure 2J). However, following the addition of $100 \mu \mathrm{M}$ AG490, a specific inhibitor of activated Jak2, to OGD-treated cells, administration of $1 \mu \mathrm{g} / \mathrm{l} \mathrm{SN}$ did not increase MAP-2-immunoreactive cell density in PCC (Figure 2N).

$S N$ administration i.v. improves neurological behavior after cerebral ischemia. To evaluate the neuroprotective effect of i.v. administration of $\mathrm{SN}$ at 30 minutes after cerebral ischemia, body asymmetry trials and locomotor activity tests were used to assess neurological behavior in SN-treated and control stroke rats ( $n=10$ per group). From 14 to 28 days after treatment, rats treated with SN exhibited significantly reduced body asymmetry compared with control rats (Figure $3 \mathrm{~A}$ ). Locomotor activity such as vertical activity, vertical movement time, and number of vertical movements significantly increased in rats receiving $\mathrm{SN}$ treatment compared with control 


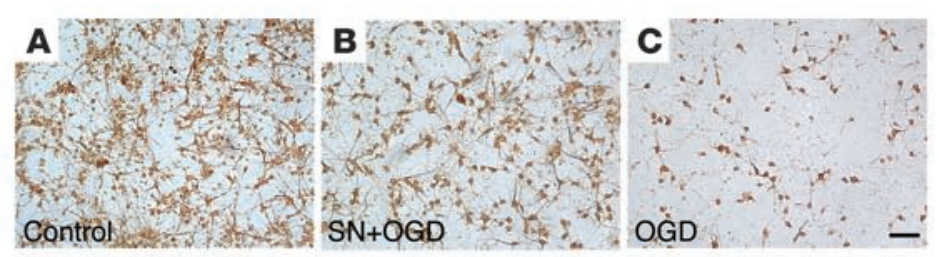

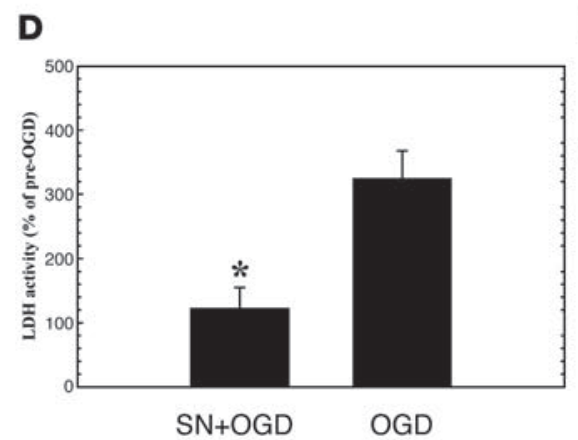

G

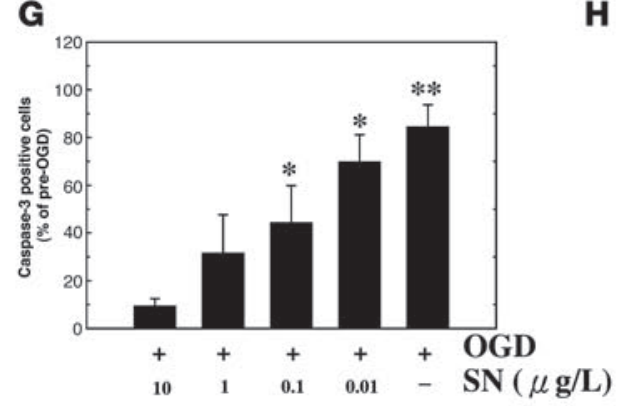

E

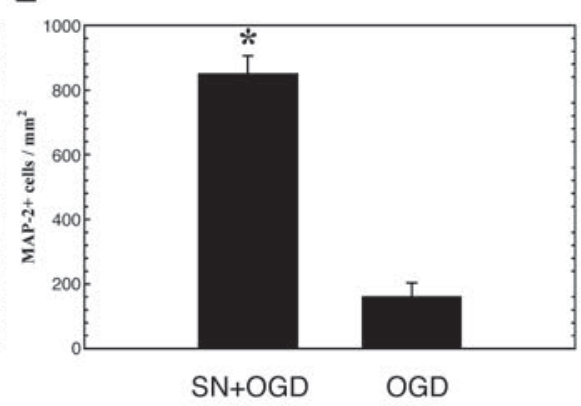

H

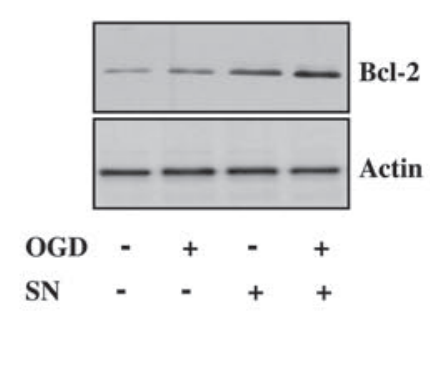

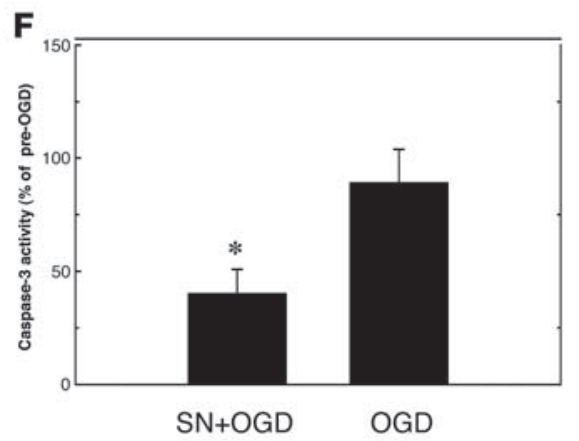
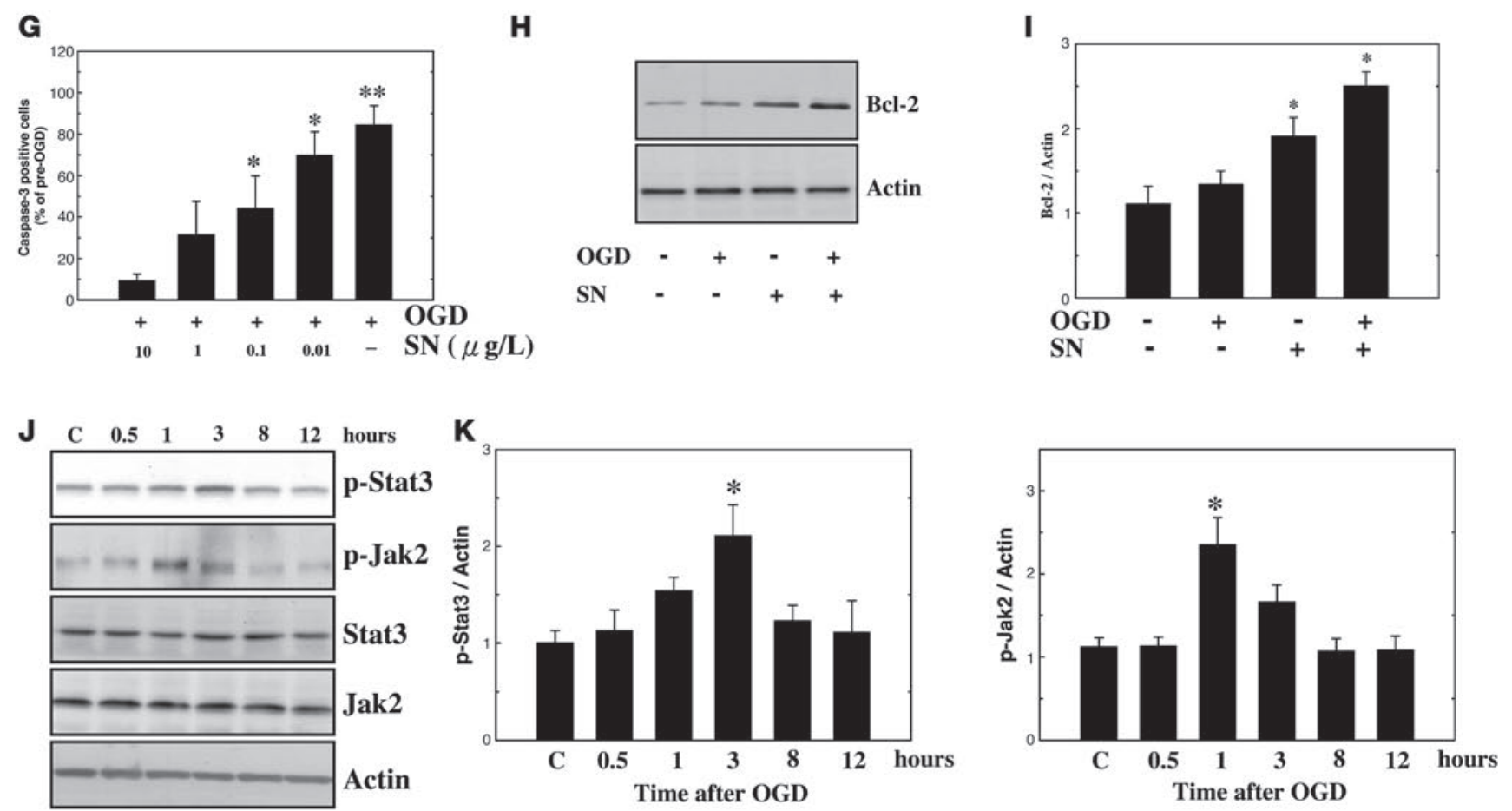

L

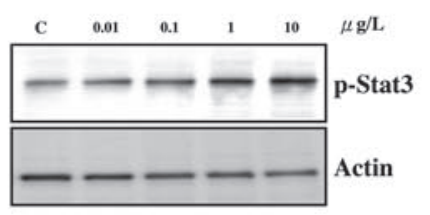

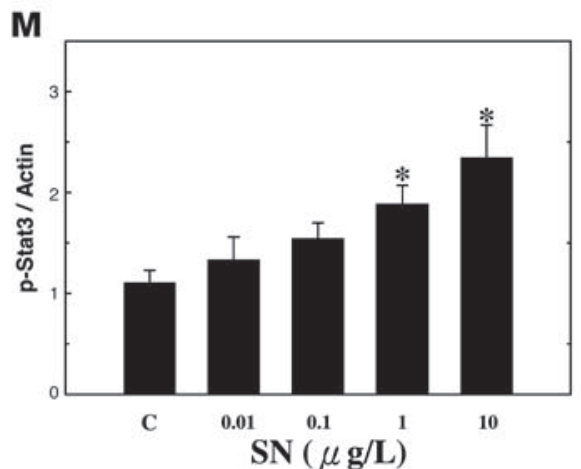

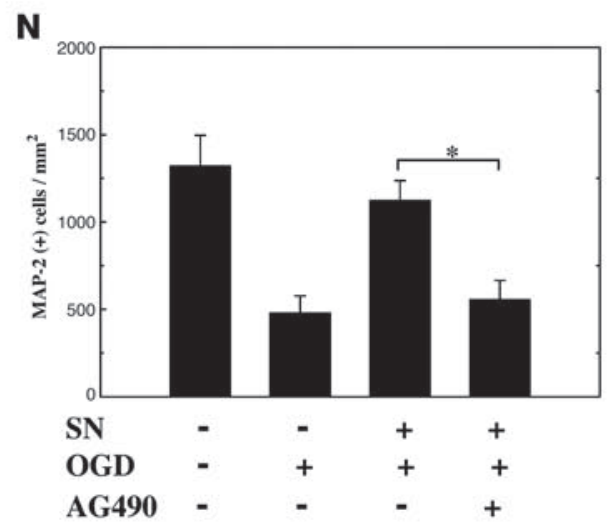




\section{Figure 2}

Pretreatment with SN attenuates OGD-induced toxicity through a specific signaling pathway in PCCs. (A-C) Representative microscopic morphology of PCCs under various conditions (control, SN plus OGD, and OGD alone). (D) Cell death assessed by LDH activity in media of PCC cultures with or without $1 \mu \mathrm{g} / \mathrm{I} \mathrm{SN}$ before exposure to OGD. SN pretreatment significantly attenuated OGD-induced cell death. (E) The density of MAP-2-immunreactive PCCs was significantly reduced by OGD, but returned to control levels with SN pretreatment. (F) Fluorimetric measurement under OGD. A significant reduction of caspase-3 activity was seen in the SN-pretreated PCCs. (G) A significant reduction of caspase-3+ immunofluorescent cells was observed in the SN-treated group. (H and I) Quantitation of Western blot in PCCs treated with SN under OGD showed significantly increased Bcl-2 expression. (J) Expression of p-Stat3, Stat3, p-Jak2, and Jak2. (K-M) The ratio of p-Stat3 and p-Jak2 to actin protein after SN treatment peaked at about a 2-fold increase in treated cells compared with control cells in a time- and dose-dependent manner. There was no statistically significant difference in Stat3 and Jak2 levels between treated and control cells. (N) Addition of AG490 to OGD-treated cells significantly reduced MAP-2-immunoreactive cell density in PCCs. Data are mean \pm SEM. ${ }^{\star} P<0.05,{ }^{* \star} P<0.01$ vs. control. Scale bar: $50 \mu$ m.

animals between 14 and 28 days after cerebral ischemia (Figure 3 , B-D). Furthermore, measurement of improvement in grip strength was performed to examine the fore-limb strength for all experimental rats before treatment and at 28 days after each of the 2 treatments. A higher percentage of improvement in grip strength was found in the SN-treated group compared with the control group (Figure 3E). However, administration of AG490 or another Jak2 inhibitor, TG101209, to the SN-treated rats ( $n=8$ per group) blocked the recovery of all behavior modalities from neurological dysfunction after cerebral ischemia (Figure 3, A-E).

SN administration i.v. reduces infarct volume after cerebral infarction. To sequentially observe and quantify the volume of cerebral infarction without sacrifice of the experimental rats, MRI of 16 rats $(n=8$ per group) showing uniform cortical infarctions was performed 1-28 days after the induction of cerebral ischemia. Cortical infarcts in rats treated with $\mathrm{SN}$ showed remarkable size reductions from day 7 to day 28 (Figure 3F). By contrast, cortical infarcts in control rats showed only a small decrease in size over the same time period (Figure 3G).

The 8 rats that underwent SN treatment at 30 minutes after cerebral ischemia showed mild infarction after cerebral ischemia. At 7 days after cerebral ischemia, the infarct volume was significantly less in SN-treated rats than saline-treated controls $\left(73 \pm 17 \mathrm{~mm}^{3}\right.$ vs. $182 \pm 16 \mathrm{~mm}^{3}$; Figure $\left.3 \mathrm{H}\right)$. The area of largest infarction was significantly less in SN-treated rats than in control rats $\left(9.4 \pm 3.3 \mathrm{~mm}^{2}\right.$ vs. $19.7 \pm 2.9 \mathrm{~mm}^{2}$; Figure $3 \mathrm{H}$ ). Infarcted slices were also significantly less in SN-treated animals than in control animals $(3.1 \pm 0.5$ slices/rat vs. $6.7 \pm 0.4$ slices/rat; Figure $3 \mathrm{H}$ ).

Enhancement of glucose metabolic activity in $\mathrm{SN}$-treated stroke rats. To verify whether i.v. SN administration enhances glucose metabolic activity, each experimental rat was examined by $\left[{ }^{18} \mathrm{~F}\right]$ fluoro-2-deoxyglucose-PET ( ${ }^{18}$ FDG-PET). Glucose metabolism was measured by ${ }^{18}$ FDG-microPET 1 week after each treatment. The microPET image showed a striking increase of ${ }^{18} \mathrm{FDG}$ uptake over the right cortex of the SN-treated group (Figure 3I). Semiquantitative measurement of relative glucose metabolic activity of the right hemisphere relative to the nonstroke hemisphere revealed significant enhancement in the SN-treated rats compared with controls $(n=8$ per group; Figure 3, I and J).

SN does not influence physiological parameters. To demonstrate that the neuroprotective effect of SN did not occur as a result of changes to other physiological parameters, systemic physiological parameters were analyzed in 14 experimental rats at 1.5 hours after SN administration. Compared with vehicle control, i.v. administration of SN did not alter systemic blood pressure, blood gases, blood glucose, or serum electrolyte levels ( $n=7$ per group; Table 1 ).

$S N$ protects penumbric neurons from cerebral ischemic damage. To verify the neuroprotective effect of $\mathrm{SN}$ in reducing neuronal injury in the penumbral region after cerebral ischemia (Figure 4, A and B), we ana- lyzed ischemic brain tissues for neuronal survival using specific antibodies that recognize neuron-specific proteins (Neu-N conjugated with FITC and MAP-2 with Cy3; Figure 4, C-N). In the penumbral region surrounding the ischemic cores, the number of $\mathrm{MAP}-2^{+}$and Neu- $\mathrm{N}^{+}$cells significantly increased in SN-treated rats $(n=8$; Figure $4, \mathrm{D}$ and $\mathrm{J}$ ) compared with vehicle control rats $(n=8$; Figure $4, \mathrm{G}$ and $\mathrm{M})$. At 2 days after cerebral ischemia, ischemic brain tissue from animals injected with vehicle contained fewer MAP- $2^{+}$or Neu- $\mathrm{N}^{+}$cells in the penumbral region than did that of SN-treated rats (Figure 4O).

$S N$ rescues neural tissue by blocking caspase-3 activity and increasing expression of antiapoptotic and signal-transduction protein. In order to study the neuroprotective mechanism of SN in vivo, 12 rats ( $n=6$ per group) were euthanized 8 hours after MCA ligation and subjected to caspase- 3 immunoreactivity tests. The penumbra surrounding the ischemic cores of the $\mathrm{SN}$-treated rats only contained a few cells expressing activated caspase-3. Ischemic brain tissue from animals injected with the vehicle control, however, contained many cells positive for activated caspase- 3 in both the penumbra and the ischemic core. Quantitatively, rats treated with SN showed fewer cells positive for activated caspase-3 after ischemia compared with controls (data not shown).

To investigate the molecular mechanism underlying the antiapoptotic effect of SN, we examined the expression of apoptosisrelated proteins and signal transduction proteins. Western blot showed significantly upregulated expression of antiapoptotic proteins such as Bcl-2 and Bcl-xL in SN-treated rats at 24 hours after cerebral ischemia compared with control rats (Figure 4, P and Q). In addition, significantly increased expression of phosphorylated Stat3 (p-Stat3) was found in the SN-treated ischemic rats compared with the vehicle control (Figure 4R). However, the increased expression of p-Stat 3 was abolished by AG490 or TG101209 in the SN-treated rats (Figure 4R). Finally, the reduction of infarct volume after $\mathrm{SN}$ administration was also blocked by injection of AG490 or TG101209 (Figure 5, A and B). These data suggest that the antiapoptotic mechanism in cerebral ischemia related to SN treatment might involve inhibition of caspase-3 and upregulation of $\mathrm{Bcl}-2, \mathrm{Bcl}-\mathrm{xL}$, and $\mathrm{p}-\mathrm{Stat} 3$ expression.

$S N$ protects neural tissues from apoptosis. Cellular apoptosis in ischemic rat brain was studied in SN-treated and control rats $(n=6$ per group) using TUNEL analysis. The control animals, which did not receive MCA ligation, showed almost no TUNEL staining in their brain sections. The penumbral region surrounding the ischemic cores of SN-treated rats contained fewer TUNEL-positive cells than did that of vehicle controls (Figure 5C). Semiquantitatively, animals treated with vehicle had significantly more TUNEL-positive cells than those treated with SN (Figure 5C). TUNEL-positive cells were distributed mainly in the ischemic core of the cerebral cortex, with the labeling essentially found in the nucleus of neuronal cells. 
B
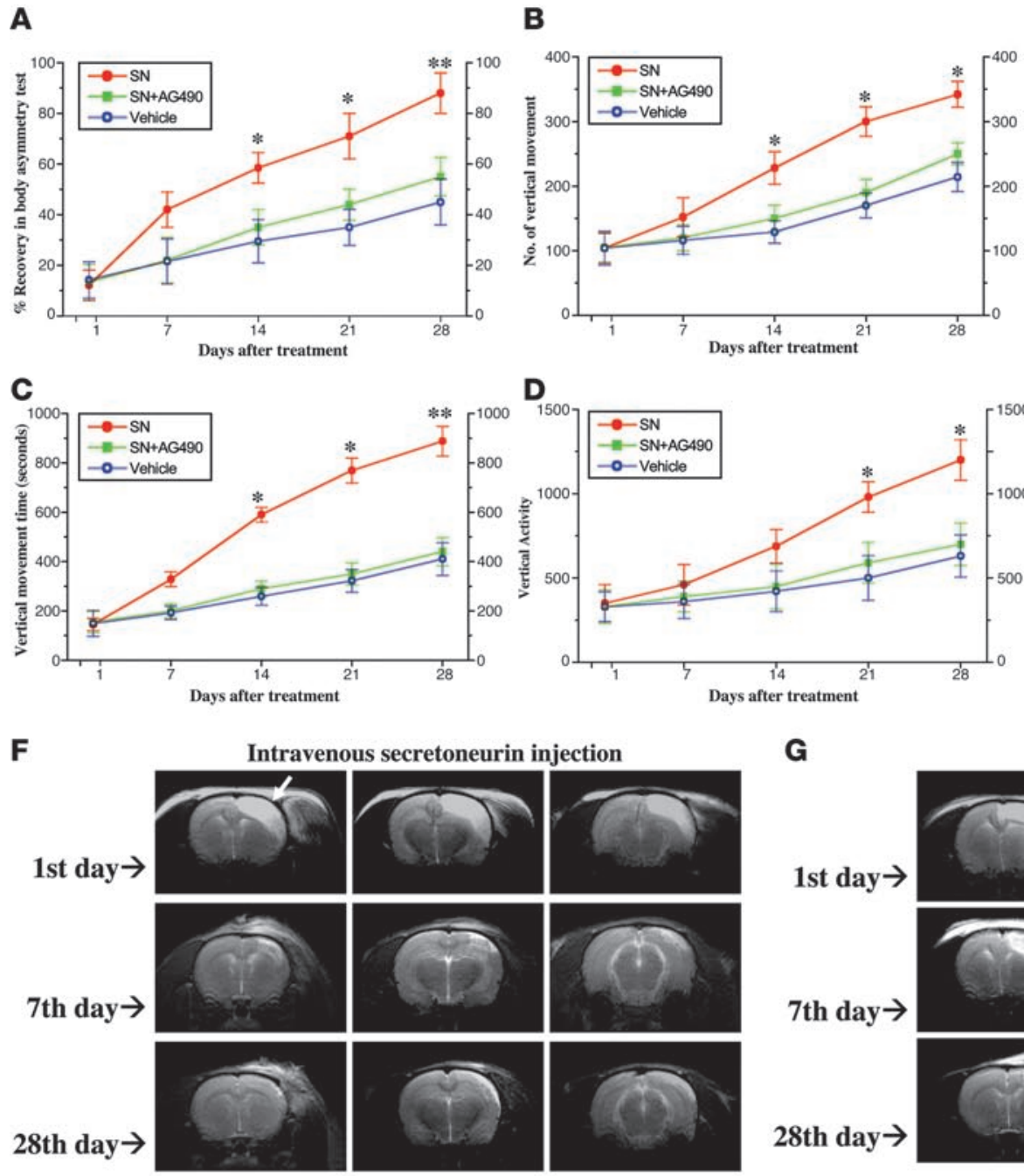

H

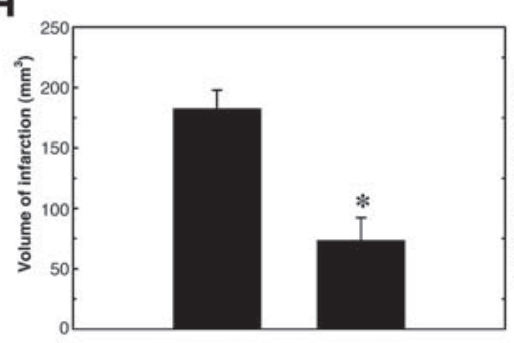

Control SN

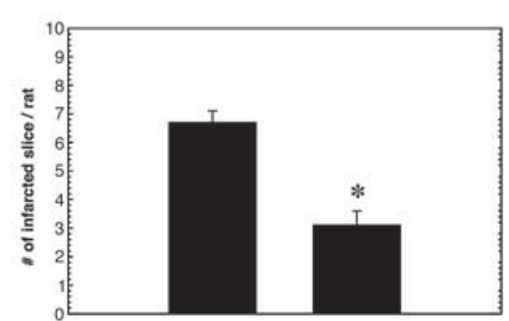

Control SN
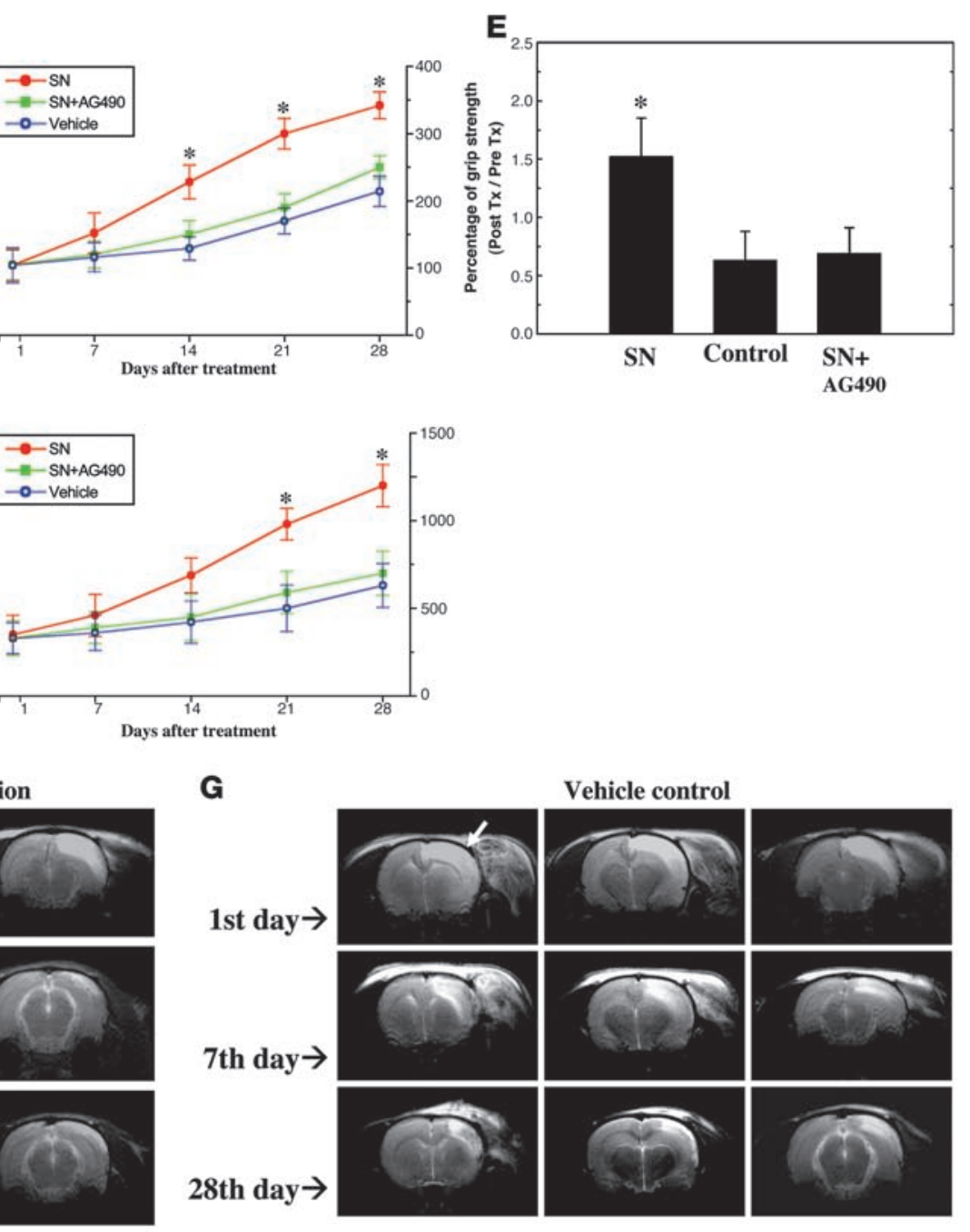

I

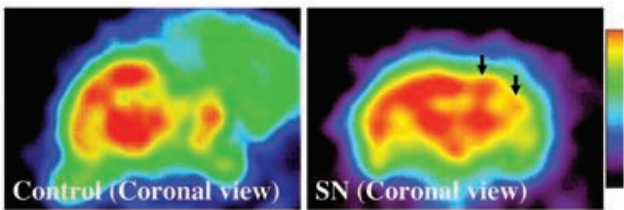

Control SN

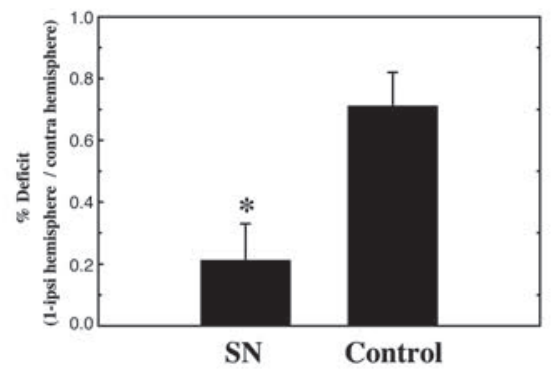




\section{Figure 3}

SN administration i.v. in cerebral ischemic rats improves neurological dysfunction and reduces infarct size. (A) From 14 to 28 days after treatment, ischemic rats receiving SN i.v. showed significantly reduced body asymmetry after MCA ligation compared with controls. (B-D) Rats receiving SN showed significantly increased locomotor activities 14-28 days after treatment. (E) Final results of grip strength measurements showed a higher grip strength ratio in the SN-treated rats than in control rats. (F and $\mathbf{G}$ ) Representative images of ischemic brain $\mathrm{MRI}$; the white areas (white arrows) are the infarcted zones in the right cerebral cortices of the SN-treated and control rats on days 1, 7, and 28 after cerebral infarction. (H) Quantitation of the infarct volume showed significant reduction in rats treated with SN on the seventh day after cerebral ischemia compared with controls. The area of largest infarction in the ischemic brain and the number of infarcted sections per rat were also reduced by SN treatment. (I) Representative ${ }^{18}$ FDG-PET (coronal view) of the right cortices (black arrows) of SN-treated and control rats. (J) Semiquantitative measurement showed that relative glucose metabolic activity in the right cortex, shown as the ratio of the ispilateral hemisphere to the contralateral hemisphere, was much greater in the SN-treated group than in the control group. Data are mean \pm SEM. ${ }^{\star} P<0.05,{ }^{* *} P<0.01$ vs. control.

SN enhances stem cell mobilization and homing to brain. BrdU labeling and analysis by transgenic GFP-chimeric mice were used to demonstrate the homing and engraftment of intrinsic neural progenitor cells (INPCs) and BMSCs to the brain. Seven days after cerebral ischemia, in SN-treated rats $(n=6)$, cumulative BrdU labeling revealed a few BrdU-immunoreactive cells in the ipsilateral cortex near the infarct boundary (Figure 6, A-D) and subventricular region of ischemic hemisphere (Figure 6, E-H). BrdU-immunoreactive cells were also found around the lumen of varying calibers of blood vessels in the perivascular portion of the ischemic hemisphere (Figure 6, I-L). BrdU pulse labeling showed significantly more BrdU-immunoreactive cells in the penumbral region in SN-treated rats than saline control rats $(n=8$ per group; Figure $6 \mathrm{M})$. Moreover, in transgenic GFP-chimeric mice, a significant increase of $\mathrm{GFP}^{+}$cells (showing green fluorescence) was observed in the penumbral region of SNtreated mice compared with controls (Figure $6 \mathrm{~N}$ ).

$S N$ promotes engrafted stem cells to differentiate into neural cells in vivo. To determine whether INPCs and BMSCs differentiate into neuronal and glial cells at ischemic sites in the brains of SN-treated mice, 3D immunofluorescent colocalization experiments were performed on each brain slice from SN-treated and control mice. Many $\mathrm{GFP}^{+}$ cells were dispersed over the bilateral striatum, hippocampus, and penumbral region, which were costained with the specific markers Neu-N, glial fibrillary acidic protein (GFAP), and MAP-2 (Figure 7A). In addition, the hippocampal, subventricular, and ischemic penumbral regions of SN-treated rats revealed an increase in $\mathrm{BrdU}^{+}$ cells coexpressing the neural phenotypes of $\mathrm{GFAP}^{+}$and Neu-N ${ }^{+}$cells (Figure 7B). Furthermore, SN-treated rats revealed an increase in $\mathrm{GFP}^{+}$Musashi- $1^{+} \mathrm{BrdU}^{+}$and $\mathrm{GFP}^{+} \mathrm{GFAP}^{+} \mathrm{BrdU}^{+}$in the hippocampal, subventricular, and ischemic penumbral regions (Figure $7 \mathrm{C}$ ).

$\mathrm{SN}$ induces angiogenesis in vivo. To determine whether $\mathrm{SN}$ induces angiogenesis through proliferation of endothelial cells and homing INPCs and BMSCs differentiating into vascular-endothelial cells at ischemic sites, double-staining IHC, FITC-dextran perfusion, and blood vessel density assays were performed on each brain slice from $\mathrm{SN}$-treated and control mice. There were several $\mathrm{BrdU}^{+}$and $\mathrm{GFP}^{+}$ cells with vascular phenotypes ( $\mathrm{vWF}^{+}$cells) around the perivascular and endothelial regions (Figure 8A) of the ischemic hemispheres of $\mathrm{SN}$-treated rats. SN-treated rats showed much more enhanced cerebral microvascular perfusion with FITC-dextran than did controls ( $n=6$ per group; Figure 8, B and C). Quantitative measurement of blood vessel density examined by CD31 immunoreactivity (Figure 8 , D and E) showed that ischemic rats treated with SN had significantly increased neovasculature in the penumbral region compared with controls ( $n=6$ per group; Figure $8 \mathrm{~F}$ ).

$S N$ facilitates cerebral blood flow in the ischemic brain. In order to determine whether increased blood vessel density enhances functional cerebral blood flow $(\mathrm{CBF})$ in the ischemic brain, experimental rats were monitored by laser Doppler flowmetry under anesthesia after cerebral ischemia. At 1 week after cerebral ischemia, there was a significant increase in CBF in the MCA cortex of the SN-treated rats compared with controls ( $n=6$ per group; Figure $8 \mathrm{G}$ ).

\section{Discussion}

In this study, we demonstrated that $\mathrm{SN}$ exerted a neuroprotective role in vitro by reducing injury-induced LDH activity, blocking the activation of the downstream apoptotic enzyme caspase-3, and upregulating the antiapoptotic proteins $\mathrm{Bcl}-2$ and $\mathrm{Bcl}-\mathrm{xL}$. We also showed that $\mathrm{SN}$ treatment at 30 minutes after cerebral ischemia in rats significantly blocked caspase- 3 activity induced by ischemia-mediated apoptosis and also enhanced neural repair to reduce infarct volume and neurological dysfunction. Furthermore, in our study SN was neuroprotective in PCC cultures and in ischemic rats by activating the Jak2/Stat 3 signal transduction pathway, an effect that was blocked by the specific inhibitor AG490. Besides the neuroprotective effect of SN, we also verified an increase in mobilization of endogenous stem cells to ischemic areas of the brain, which was demonstrated in transgenic GFPchimeric mice. This increased endogenous stem cell mobilization and homing to the ischemic brain enhanced neovascularization and neurogenesis over the peri-infarcted area, resulting in stimulation of cellular repair in the penumbra of the ischemic brain. In conclusion, the preservation of cells immunoreactive for MAP-2 and Neu-N and the greater promotion of stem cell differentiation in the penumbra of the ischemic brain compared with the control suggest that $\mathrm{SN}$ exerts a neuroplastic effect on neurons in the adult rat brain after stroke.

\section{Table 1}

Physiological parameters were not altered by SN

\begin{tabular}{lccc} 
Parameter & $\mathbf{S N}(\boldsymbol{n}=7)$ & Control $(\boldsymbol{n}=7)$ & $\boldsymbol{P}$ \\
$\mathrm{pH}$ & $7.38 \pm 0.041$ & $7.33 \pm 0.03$ & 0.91 \\
$\mathrm{PaCO}_{2}(\mathrm{mmHg})$ & $47.03 \pm 1.11$ & $51.4 \pm 2.23$ & 0.26 \\
$\mathrm{PaO}_{2}(\mathrm{mmHg})$ & $89.7 \pm 3.15$ & $94.17 \pm 3.0$ & 0.23 \\
$\mathrm{HCO}_{3}^{-}\left(10^{-3} \mathrm{~mol} / \mathrm{l}\right)$ & $28.3 \pm 1.56$ & $24.8 \pm 1.61$ & 0.43 \\
$\left.\mathrm{Hematocrit}^{\circ} \%\right)$ & $45.13 \pm 2.3$ & $43.1 \pm 3.21$ & 0.28 \\
$\mathrm{Hemoglobin}^{+}(10 \mathrm{~g} / \mathrm{l})$ & $14.3 \pm 0.61$ & $15.4 \pm 0.66$ & 0.27 \\
$\mathrm{Na}^{+}\left(10^{-3} \mathrm{~mol} / \mathrm{l}\right)$ & $138.4 \pm 4.5$ & $143.5 \pm 2.3$ & 0.65 \\
$\mathrm{~K}^{+}\left(10^{-3} \mathrm{~mol} / \mathrm{l}\right)$ & $4.1 \pm 0.26$ & $4.7 \pm 0.42$ & 0.79 \\
$\mathrm{Ca}^{+}\left(10^{-2} \mathrm{~g} / \mathrm{l}\right)$ & $4.1 \pm 0.41$ & $3.72 \pm 1.2$ & 0.54 \\
$\mathrm{Glucose}\left(10^{-2} \mathrm{~g} / \mathrm{l}\right)$ & $149.4 \pm 28.5$ & $143.1 \pm 15.2$ & 0.53 \\
$\mathrm{MBP}(\mathrm{mmHg})$ & $78.9 \pm 8.2$ & $81.3 \pm 6.4$ & 0.55 \\
$\mathrm{HR}(\mathrm{bpm})$ & $391 \pm 25$ & $404 \pm 19$ & 0.66 \\
\hline
\end{tabular}

MBP, mean blood pressure; HR, heart rate. $P$ values were determined by 2 -tailed Student's $t$ test. 
A

$\circ \quad \square \Delta$

Secretoneurin
B

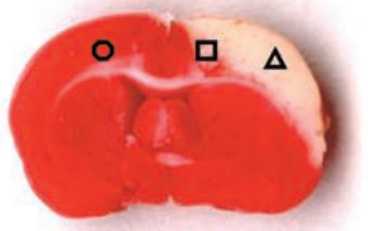

Control
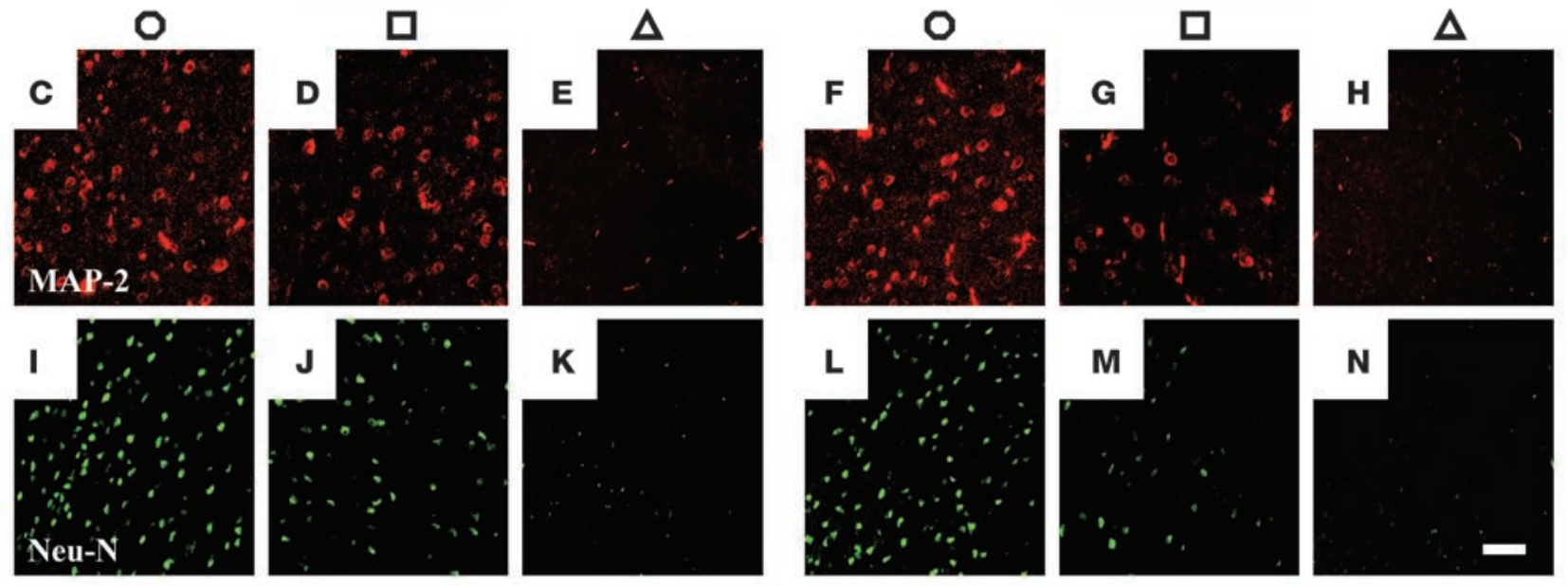

O
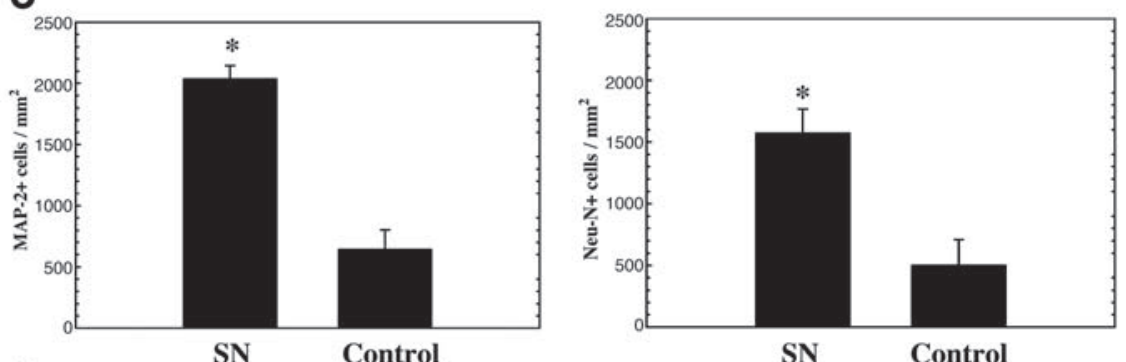

$\mathbf{P}$
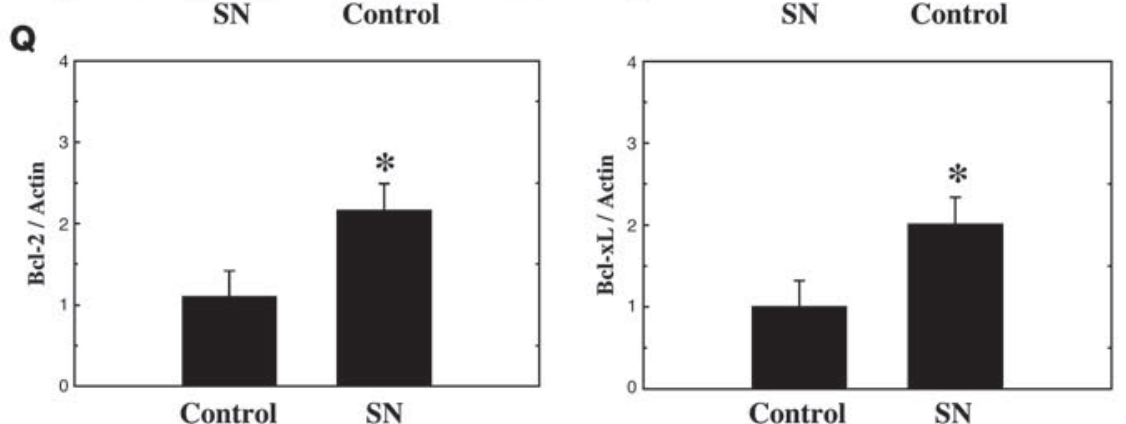

R
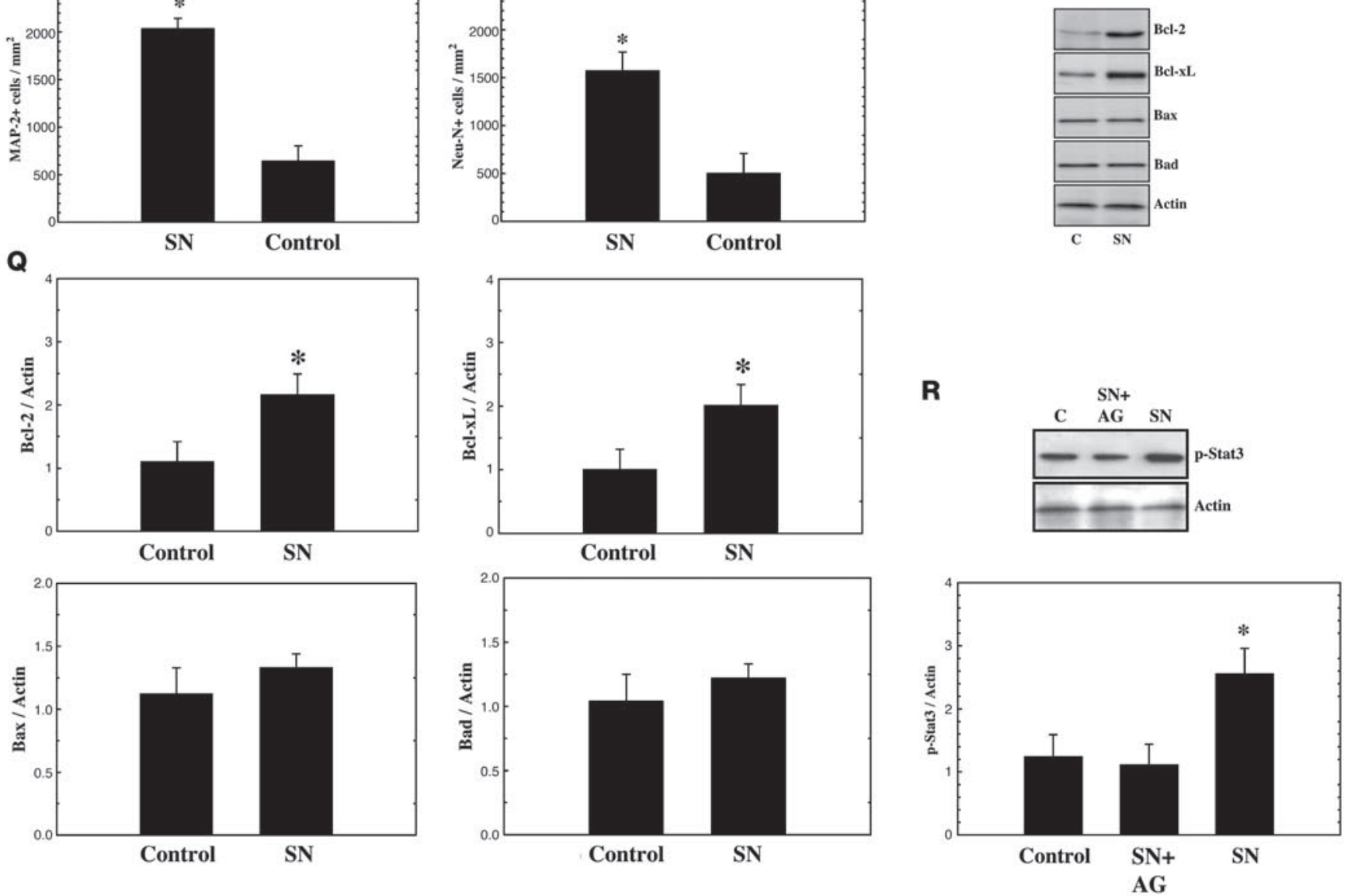
Figure 4

SN administration i.v. 30 minutes after cerebral ischemia rescued neurons from ischemic injury by directly inhibiting apoptotic signals. (A and B) Representative triphenyltetrazolium chloride-stained brain slices from rats injected with $\mathrm{SN}$ and vehicle. (C-N) Sections from the brain slices in $\mathbf{A}$ and $\mathbf{B}$ were immunostained to identify MAP-2+ neurons $(\mathbf{C}-\mathbf{H})$ and Neu- $\mathrm{N}^{+}$neurons $(\mathbf{I}-\mathbf{N})$ in 3 different regions (corresponding with symbols in $\mathbf{A}$ and B). (O) The number of MAP-2- and Neu-N-immunoreactive cells in the penumbral area (shown in $\mathbf{D}, \mathbf{G}, \mathbf{J}$, and $\mathbf{M}$ ) was significantly increased in SN-treated rats compared with control rats. (P and $\mathbf{Q})$ Significantly increased expression of $\mathrm{Bcl}-2$ and $\mathrm{Bcl}$ - $\mathrm{xL}$ in $\mathrm{SN}$-treated rats. (R) Increased expression of $\mathrm{p}$-Stat3 in SN-treated rats, which was knocked down by AG490. Data are mean \pm SEM. ${ }^{*} P<0.05$ vs. control. Scale bar: $40 \mu \mathrm{m}$.

In agreement with previous studies, we found $\mathrm{SN}$ to have functions similar to those of glial cell-derived neurotrophic factor (GDNF), correlated to growth $(18,22-24)$ and repair $(6,25,26)$ of injured neural tissue. Some literature has indicated that expression of $\mathrm{SN}$ is upregulated after cerebral ischemic insult (7). Similarly, TGF- $\beta 1$ and GDNF mRNA levels have also been shown to increase after brain injury $(27,28)$. From this evidence, investigators have suggested that endogenous protective mechanisms could be activated after such insults $(27,28)$. In the present study, we have shown that increased expression of $\mathrm{SN}$ in the human stroke brain and in cerebral ischemic rats was induced to prevent further damage through endogenous genetic modulation following neuronal injury. Some reports have also demonstrated that a significant loss of SN-IR is found in the hippocampi of patients with Alzheimer disease and that a large part of the neuritic plaques in these patients are $\mathrm{SN}$-immunopositive; the authors concluded that expression of SN in the hippocampus was closely correlated with synaptic loss in Alzheimer disease $(3,5)$.

In previous reports, $\mathrm{SN}$ has been considered to be an angiogenic cytokine like VEGF, which has mitogenic and antiapoptotic effects on endothelial cells $(18,25)$. These reports demonstrated that SN acts as a survival agent, as demonstrated by the reduction of annexin $\mathrm{V}$-positive cells under serum starvation in HUVECs. In addition, these reports indicated that the mitogenic signaling pathway with $\mathrm{SN}$ is through activation of ERK1/2 and Akt pathways (18).

A

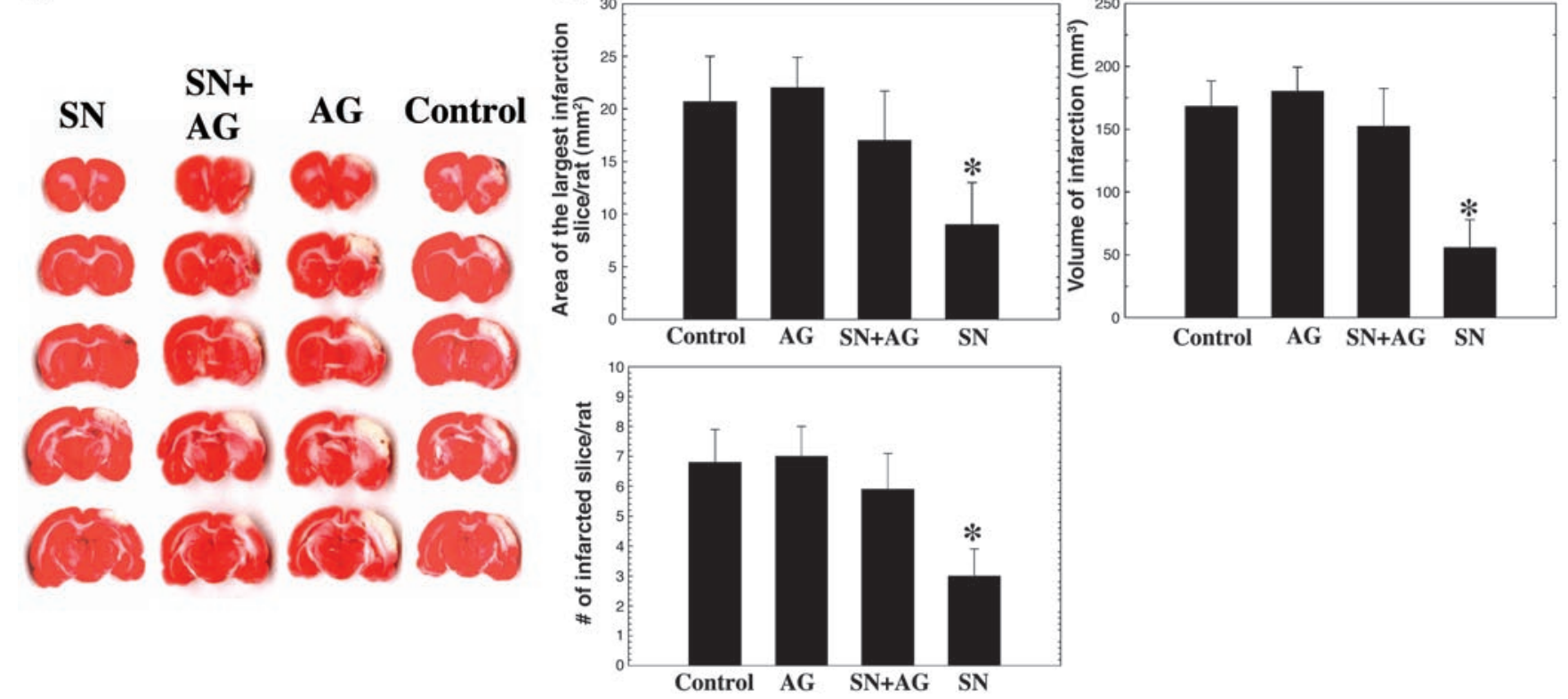

C
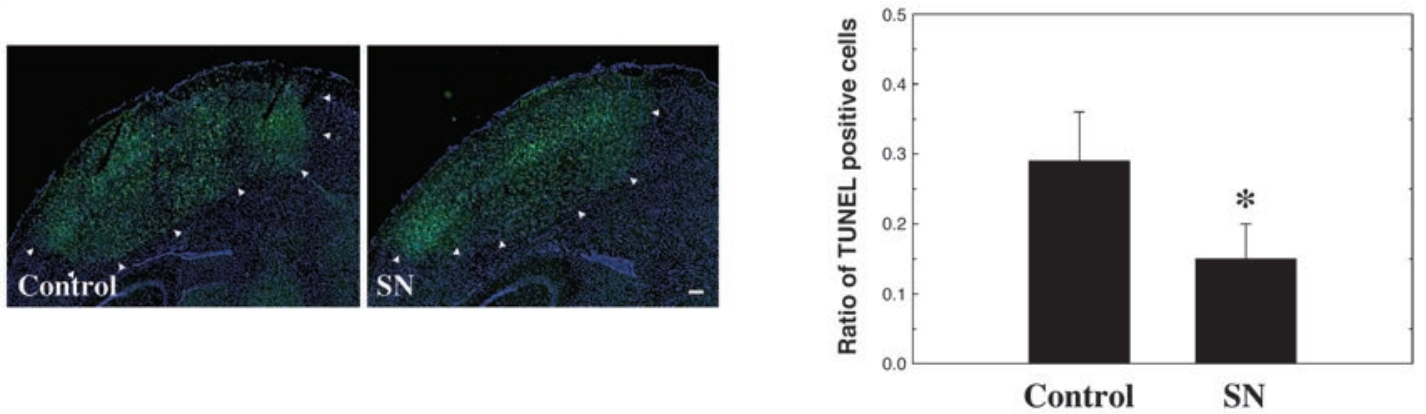

Figure 5

SN reduces apoptosis by activating the Jak2/Stat3 pathway. (A and B) Two days after cerebral ischemia, the SN-induced reduction of infarct volume was blocked by infusion of the inhibitor AG490. (C) Representative image of TUNEL (green) and Hoechst 33342 (blue) costaining showing cell death in penumbrae of ischemic brains (arrowheads) from saline control and SN-treated rats. There were significantly fewer TUNEL-positive cells in SN-treated brain compared with saline control. Data are mean \pm SEM. ${ }^{*} P<0.05$ vs. control. Scale bar: $40 \mu \mathrm{m}$. 

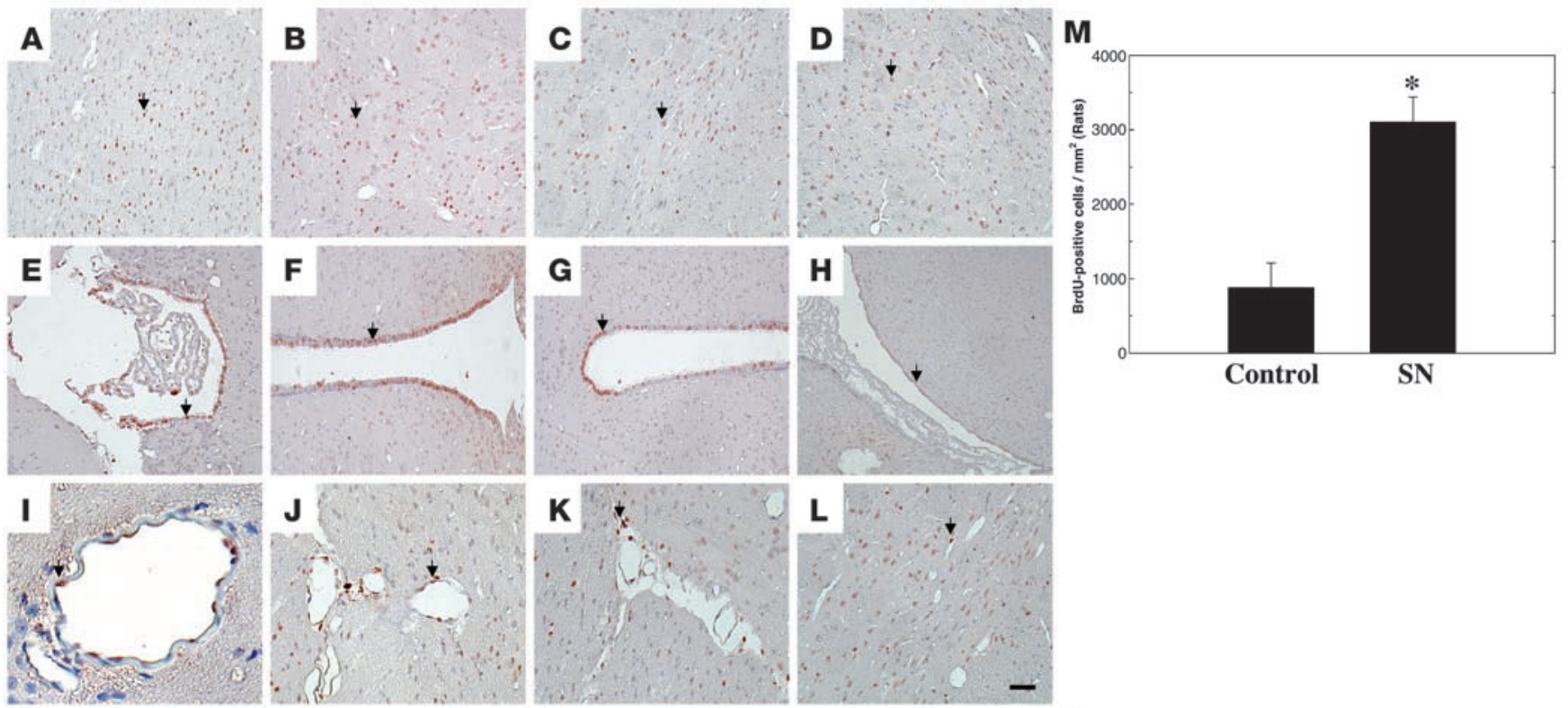

$\mathbf{N}$
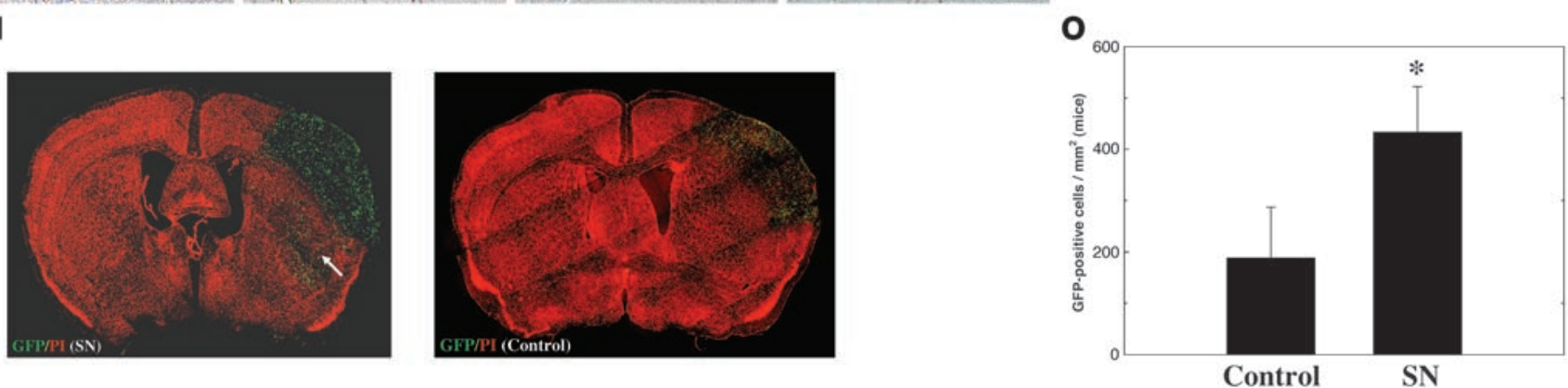

Figure 6

SN administration i.v. enhanced the mobilization of stem cells in rats and mice. (A-L) In SN-treated rats 30 minutes after cerebral ischemia, a few BrdU-immunoreactive cells (arrows) were detected in the ipsilateral cortex near the infarct boundary (A-D) and subventricular area (E-H). (I-L) BrdU-immunoreactive cells were also found around blood vessels in the ipsilateral cortex. (M) Quantitative analysis revealed that the number of BrdU-immunoreactive cells significantly increased in the ipsilateral hemisphere of SN-treated rats compared with control rats. ( $\mathbf{N}$ and $\mathbf{O}$ ) Representative constructive images of IHC in GFP-chimeric mice showed significantly increased GFP+ cells over the peri-infarcted and striatal areas (white arrow) in the SN-treated mice compared with controls. Original magnification, $\times 30$. Data are mean $\pm \mathrm{SEM}$. ${ }^{*} P<0.05$ vs. control. Scale bar: $50 \mu \mathrm{m}$.

Although SN may exert a survival effect and induce immature cerebellar granular neurons to differentiate by promoting neurite outgrowth (6), few reports have focused on the neuroprotective role of $\mathrm{SN}$ in vitro and in vivo. We have demonstrated, for the first time to our knowledge, that $\mathrm{SN}$ exerts an antiapoptotic effect by reducing LDH activity, blocking activated caspase-3 activity, and upregulating expression of antiapoptotic proteins in primary cortical neurons in vitro. We also found that $\mathrm{SN}$ transduced an antiapoptotic signal in the activation of the Jak 2 and Stat 3 signal pathways and that inhibition of these pathways nullified the effect of $\mathrm{SN}$ in the primary cortical neurons. Endres et al. found that, in a cerebral ischemic animal model, proapoptotic mechanisms that facilitate caspase-3-mediated cell death are activated during the ischemic/reperfusion state (29). We found that SN disrupted the downstream caspase-3 apoptotic signal in the ischemic penumbra, resulting in protection of cortical neurons and diminished infarct volume. In addition, i.v. administration of $\mathrm{SN}$ also increased the expression of $\mathrm{Bcl}-2$ in primary cortical neurons and upregulated $\mathrm{Bcl}-2$ and $\mathrm{Bcl}-\mathrm{xL}$ in the penumbral region of cerebral ischemia. These in vivo effects of SN were conducted through induction of Jak2/Stat3, which was abolished by the specific inhibitors AG490 and TG101209. In summary, the neuroprotective effect of SN injection through direct inhibition of caspase-3 activation and enhancement of expression of antiapoptotic proteins (Bcl-2 and $\mathrm{Bcl}-\mathrm{xL}$ ) might be one of the more significant mechanisms involved in the rescue of injured neurons.

Taguchi et al. have recently shown that i.v. delivery of the CD34 subpopulation of human umbilical cord blood cells can enhance angiogenesis, neurogenesis, and functional recovery in an animal stroke model (30). They further demonstrated that CD34+ cells stimulated angiogenesis with specific neovascularization around the cortical degeneration. Neurogenesis was in turn stimulated by these events, with subsequent migration of neuroblasts into the newly restored cortex, where these cells matured and contributed to functional recovery. Some reports have also assumed that enhancement of angiogenesis, and then neurogenesis, have a plasticity effect on the recovery of ischemic neurological insult $(13,14)$. In addition to the plasticity effect in the nervous system $(31,32)$, cytokines of neuronal origin (such as nerve growth factors) and 
A
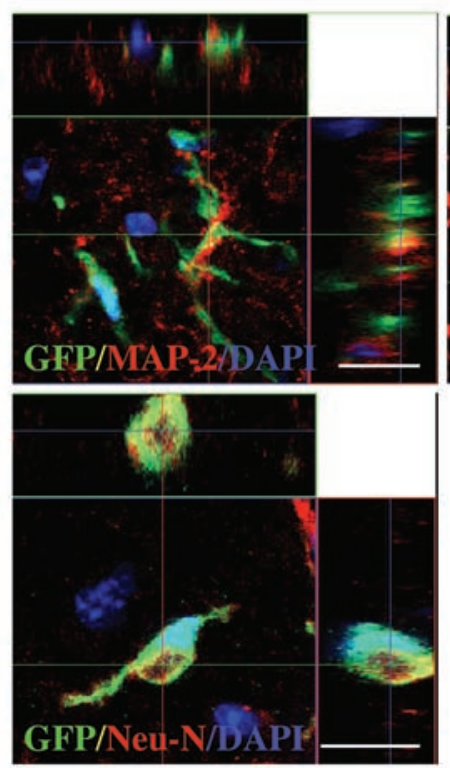

B
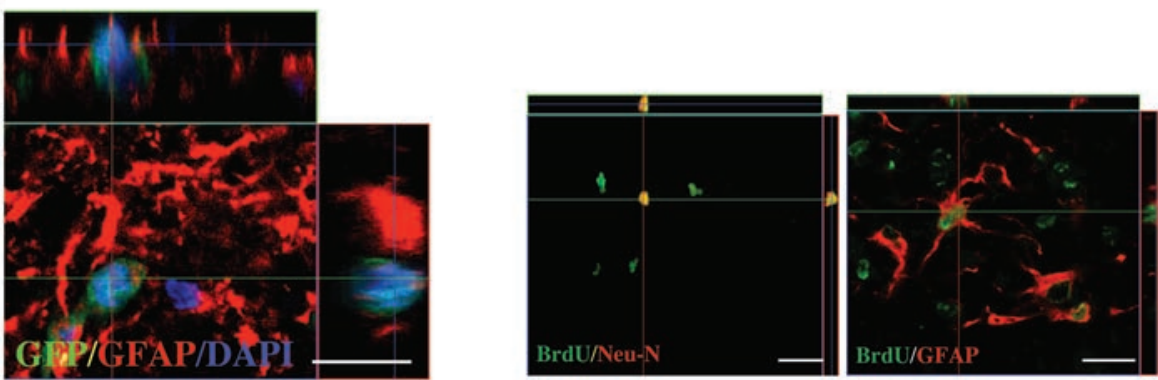

C

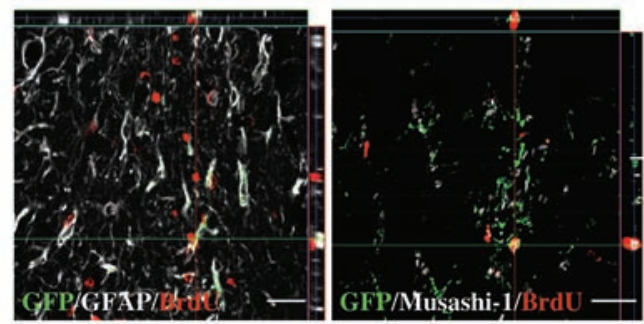

Figure 7

SN promoted the neural differentiation of engrafted stem cells as shown by laser-scanning confocal microscopy in 3D images. (A) Ischemic cortical areas of SN-treated rats revealed GFP+ cells colocalized with the neural marker of GFAP, Neu-N, and MAP-2. (B) Many BrdU+ cells colocalized with the neural markers GFAP and Neu-N were also found around the penumbric area of the ischemic hemispheres in SN-treated rats. (C) Some GFP+Musashi-1+BrdU+ and GFP+GFAP+BrdU+ cells were found in the hippocampal, penumbral, and subventricular regions. Scale bars: $50 \mu m$.

several members of the neuropeptide group (such as neuropeptide Y; ref. 33) were reported to induce angiogenesis in injured areas of the brain. Furthermore, VEGF was shown to exert neuroplastic activity (34), by enhancing vascular supply of the nerves, and to improve neural function in ischemic and diabetic neuropathy (35, 36). In the present study, we demonstrated that i.v. administration of SN 30 minutes after cerebral ischemia not only enhanced the incorporation of new vessels into the penumbral region to increase local cerebral blood perfusion, but induced neural differentiation of mobilized BMSCs and INPCs to reduce neurological dysfunction. Using a GFP-chimeric mouse model, we demonstrated that many $\mathrm{GFP}^{+}$cells (from BMSCs), especially GFP $\mathrm{Geu}^{+} \mathrm{N}^{+}$cells, migrated to the peri-infarcted area. A large amount of $\mathrm{BrdU}^{+} \mathrm{Neu}-$ $\mathrm{N}^{+}$and $\mathrm{BrdU}^{+} \mathrm{GFAP}^{+}$cells (from INPCs) were located in the subventricular and hippocampal regions in the $\mathrm{SN}$-treated mice. In addition, some $\mathrm{GFP}^{+} \mathrm{Musash}-1^{+} \mathrm{BrdU}^{+}$and $\mathrm{GFP}^{+} \mathrm{GFAP}^{+} \mathrm{BrdU}{ }^{+}$cells were also found in the hippocampal, penumbral, and subventricular regions. Functional neural regeneration and neurogenesis after SN treatment contributed to by both BMSCs and INPCs might be another important mechanism that induces improvement of neurological dysfunction following stroke. Therefore, SN may exert a significant neuroplastic effect because of efficient angiogenesis and neurogenesis in the penumbral region of the ischemic brain.

We know that circulating cytokines such as G-CSF, VEGF, stromal cell-derived factor-1 (SDF-1), statin, and erythropoietin $(17,37-40)$ enhance the mobilization of BMSCs. The mechanism of mobilization of BMSCs by these factors seems to be a receptor-mediated process involving CXCR4 and c-kit $(41,42)$, according to the SDF-1 concentration gradient theory (43). In previous studies, SN, an angiogenic cytokine, has been shown to chemoattract BMSCs, especially endothelial progenitor cells, from bone marrow to circulating peripheral blood $(18,25)$. However, the possible regulatory pathway by which SN mobilizes stem cells from bone marrow remains unknown. A previous investigation demonstrated that $\mathrm{SN}$ injection into mice caused significant proliferation of bone marrow cells positive for c-kit and Sca-1 (18). In addition, $\mathrm{SN}$ also mobilized and increased the number of Sca-1-positive cells in peripheral blood. In the present study, we demonstrated that $\mathrm{SN}$ enhanced the assembly of $\mathrm{GFP}^{+} \mathrm{vWF}^{+}$and $\mathrm{BrdU}^{+} \mathrm{vWF} \mathrm{WF}^{+}$cells (from both BMSCs and INPCs) to form many vascular lumens over the penumbral, striatal, and hippocampal regions in GFP-chimeric mice. FITC-dextran perfusion of the ischemic brain and $\mathrm{CD} 31^{+}$blood vessel density revealed considerable new vessel formation in the SN-treated mice. At 7 days after cerebral ischemia, higher cerebral blood flow was detected in the $\mathrm{SN}$-treated rats than in the controls.

In summary, we have shown that $\mathrm{SN}$ exerted neuroprotective effects in vitro by blocking activation of caspase-3, the downstream apoptotic enzyme, and upregulating the antiapoptotic proteins $\mathrm{Bcl}-2$ and Bcl-xL. It was also neuroplastic in cerebral ischemic animals by means of instigating new neuronal and vascular formation within peri-infarcted regions of brain, attenuating tissue damage and subsequently reducing infarction volume and improving neurological function. We have disclosed, for the first time to our knowledge, a neuroplastic view of SN in a hypoxia and ischemia model. We believe that the SN treatment protocol presented here holds promise for the development of new therapeutic strategies, including gene therapy and that SN may possess utility as a smallmolecular drug that can improve innate tissue regeneration and repair after cerebral ischemia to clinically significant levels.

\section{Methods}

IHC analysis of autopsy samples of human brain. We investigated autopsy brain specimens from 9 cases of fatal ischemic stroke (disease duration 
A
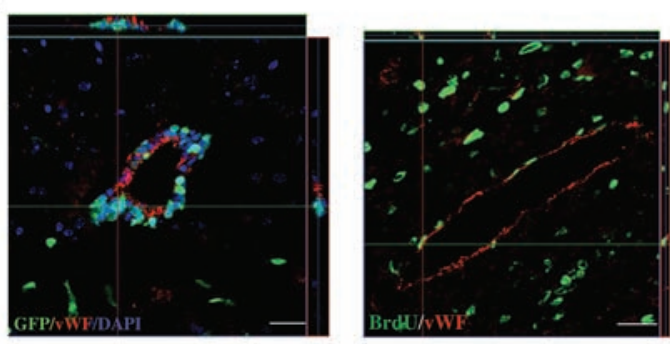

\section{$\mathbf{F}$}

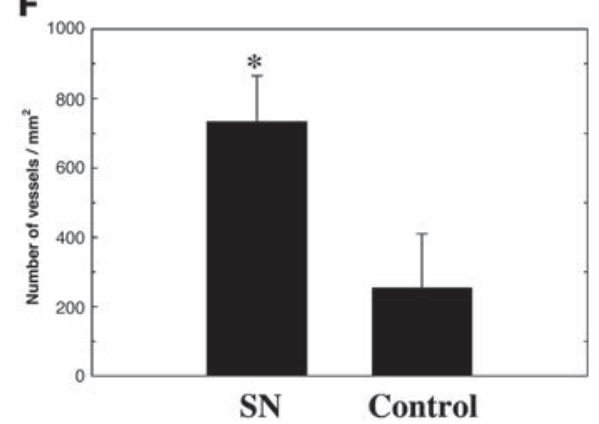

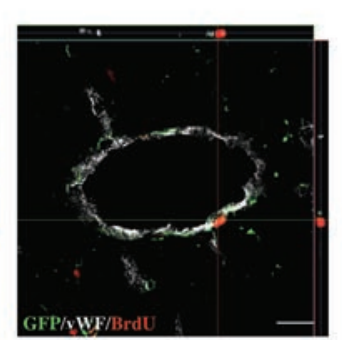
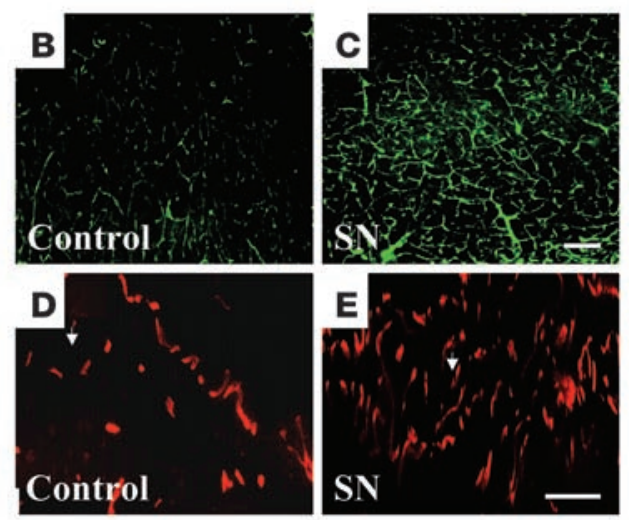

G

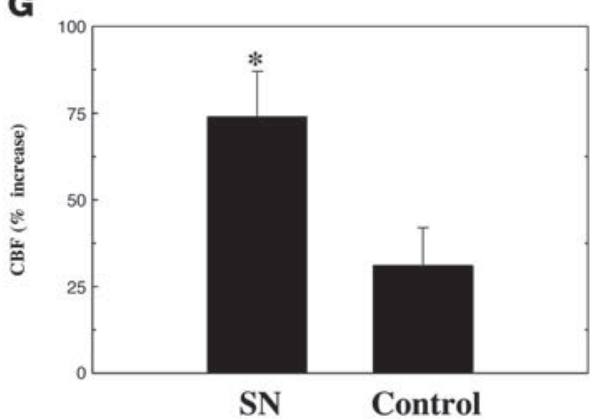

\section{Figure 8}

$\mathrm{SN}$ treatment induced angiogenesis in the ipsilateral hemisphere. (A) Colocalization analysis of GFP and BrdU immunostaining with vWF ${ }^{+}$cells around the perivascular and endothelial regions of the ischemic hemispheres of SN-treated rats. (B and C) FITC-dextran-perfused brain samples show the microvascular pattern of SN-treated and control rats. (D and E) Quantitative analysis of cerebral blood vessel density, stained by CD31 immunoreactivity (arrows), in SN-treated and control rats. (F) Ischemic rats treated with SN showed a significant increase in neovasculature in the penumbral area compared with controls. (G) Analysis of CBF using laser Doppler flowmetry monitoring 1 week after cerebral ischemia showed a significant increase in CBF of the MCA cortex of ischemic brain in the SN-treated rats compared with controls. Data are mean \pm SEM. ${ }^{\star} P<0.05$ vs. control. Scale bars: $50 \mu \mathrm{m}$.

ranged from 15 hours to 7 days) treated at the Department of Neurology of Tzu-Chi General Hospital. Autopsy surgery was performed within a mean of 8 hours after death (range, $4-12$ hours). Three patients who died of nonneurological causes served as controls (autopsies were conducted at $1,2.5$, and 4 days). The study protocol was approved by the Institutional Review Board of the Tzu-Chi General Hospital. Informed consent was obtained from relatives. Tissue sampling was based on individual infarct topography, which in each case was determined on the basis of cerebrovascular anatomy and the most recent MRI scan. On autopsy, brain areas with variable degrees of infarction were identified macroscopically, and cortical samples of about $1 \mathrm{~cm}^{3}$, including subcortical white matter, were dissected and fixed with formalin prior to embedding in paraffin or frozen at $-70^{\circ} \mathrm{C}$ until analysis, as described previously (44). SN immunostaining of brain samples was performed using specific antibody (1:200; Phoenix). Samples from corresponding areas of the contralateral or noninfarcted hemispheres and from the control brains were processed in a similar way. The extent of SN cell immunoreactivity was measured as number of cells per square millimeter.

Measurement of serum SN levels in acute stroke patients. We obtained serum samples at sequential time points (12 hours and 1, 3, 7, and 14 days) after onset of acute ischemic stroke. All patients' protocols and consents were fully reviewed and approved by the Institutional Review Board of Tzu-Chi General Hospital. Levels of SN were measured with specific antibody using indirect ELISA techniques (Phoenix). Each serum sample was run in triplicate and compared with a standard curve. All samples were assigned a random number and run without knowledge of the donor's disease or treatment status. Once the data were compiled, the sample classifications were revealed.

In vitro PCC preparation and OGD treatment. All animal research protocols were approved by the Academia Sinica Animal Care and Use Committee. PCCs were prepared from the cerebral cortex of gestation day-17 Sprague-Dawley rat embryos as previously described with modification (45). Four days after isolation, the cultures were replenished with MEM (Invitrogen) containing $0.5 \mathrm{~g} / 1 \mathrm{BSA}, 2 \%$ B27 supplement, $0.5 \mathrm{mM}$ pyruvate, and antibiotics. Finally, the culture medium was changed to serumfree neurobasal medium containing $1 \mathrm{mM}$ pyruvate, $1 \mathrm{mM}$ glutamate, $0.5 \mathrm{~g} / 1 \mathrm{BSA}, 2 \% \mathrm{~B} 27$ supplement, and antibiotics on the seventh day (45). For OGD treatment, the cells cultured with glucose-free Earle's balanced salt solution were placed for 4 hours within a hypoxic chamber (Bug Box; Ruskinn Technology) and continuously flushed with $95 \% \mathrm{~N}_{2}$ and $5 \% \mathrm{CO}_{2}$ at $37^{\circ} \mathrm{C}$ to maintain a gas-phase $\mathrm{PO}_{2}$ less than $1 \mathrm{mmHg}(\mathrm{OM}-14$ oxygen monitor; SensorMedics Corporation). Control cells were incubated in glucose-free Earle's balanced salt solution in a normoxic incubator for the same time period. OGD was terminated by switching back to normal culture conditions.

Measurement of LDH activity and immunocytochemistry of MAP-2. In order to prove the concept of neuroprotection of SN, PCCs were prepared in 24well plates and pretreated with $1 \mu \mathrm{g} / \mathrm{l} \mathrm{SN}$ (Neosystems). After 20 minutes of SN pretreatment, PCCs were subjected to OGD in the hypoxia chamber for 4 hours, and then the culture media were collected for LDH activ- 
ity assays as described previously (46). For MAP-2 immunostaining, PCC cultures were washed with PBS and fixed with $1 \%$ paraformaldehyde. The immunostaining procedure with specific antibody against MAP-2 (1:1,000; Boehringer Mannheim) and the method for quantification of MAP- $2^{+}$cell density has been described previously (47).

Measurement of activated caspase-3 activity and immunofluorescent study of caspase-3. In order to evaluate the antiapoptotic effect of SN, caspase-3 activity was measured and capase-3 IHC analysis was performed on the PCCs. PCCs were pretreated with $1 \mu \mathrm{g} / \mathrm{l} \mathrm{SN}$ and then placed in the hypoxia chamber for 2 hours. Fluorimetric assays of caspase-3 activity were performed on cells treated as described above using commercial kits (Bio-Rad) according to the manufacturer's instructions. For immunofluorescent study of activated caspase-3, primary cortical neuron cultures were treated as described above and incubated with primary antibody against active fragment of caspase-3 (R\&D Systems) as previously described (48). Caspase-3-positive cells were also quantified as previously described (48).

Total protein extraction and Western blot assay. Western blot analyses of p-Stat3, Stat3, p-Jak2, Jak2, Bcl-2, Bcl-xL, Bax, and Bad expression from PCC cultures were performed after SN treatment. Briefly, PCCs treated at a different time points $(0.5,1,3,8$, and 12 hours $)$ and with different doses $(0.01,0.1,1$ and $10 \mu \mathrm{g} / \mathrm{l})$ of SN were lysed after treatment in a buffer containing $320 \mathrm{mM}$ sucrose, $5 \mathrm{mM}$ HEPES, $1 \mu \mathrm{g} / \mathrm{ml}$ leupeptin, and $1 \mu \mathrm{g} / \mathrm{ml}$ aprotinin. Lysates were centrifuged at 13,000 $\mathrm{g}$ for $15 \mathrm{~min}$ utes. The resulting pellet was resuspended in sample buffer $(62.5 \mathrm{mM}$ Tris- $\mathrm{HCl}, 10 \%$ glycerol, $2 \%$ SDS, $0.1 \%$ bromophenol blue, and $50 \mathrm{mM}$ DTT) and subjected to SDS-polyacrylamide gel (4\%-12\%) electrophoresis. The gel was then transferred to a Hybond-P nylon membrane. This was followed by incubation with appropriately diluted antibodies to p-Stat3 (1:200; Cell Signaling), p-Jak2 (1:200; Cell Signaling), Stat3 (1:200; Santa Cruz), Jak2 (1:200; Santa Cruz), Bcl-2 (1:200; Santa Cruz), Bcl-xL (1:200; Transduction Laboratories), Bax (1:200; Santa Cruz), Bad (1:200; Transduction Laboratories), and $\beta$-actin (1:2,000; Santa Cruz). Specific Jak2 pathway inhibitor AG490 (100 $\mu \mathrm{M}$; Calbiochem), which was used to pretreat cells, was applied to the PCCs to suppress enzyme binding in order to block the transcriptional signal of Jak2 as previously described (49). Membrane blocking, primary and secondary antibody incubations, and chemiluminescence reactions were conducted for each antibody individually according to the manufacturer's protocol. The intensity of each band was measured using a Kodak Digital Science 1D Image Analysis System (Eastman Kodak).

In vivo brain ischemia/reperfusion. Adult male Sprague-Dawley rats (250-300 g) were used for this study. The rats were anesthetized with chloral hydrate $(0.4 \mathrm{~g} / \mathrm{kg}$ i.p.) and subjected to right MCA ligation and bilateral common carotid artery (CCA) clamping as previously described (50). Briefly, the bilateral CCAs were clamped with nontraumatic arterial clips. Using a surgical microscope, a $2-\mathrm{mm} \times 2$ - $\mathrm{mm}$ craniotomy was drilled at the point where the zygoma fuses to the squamosal bone, the right MCA was then ligated with a 10-0 nylon suture. Cortical blood flow was measured continuously with a laser Doppler flowmeter (PF-5010, Periflux system; Perimed AB) in anesthetized animals. A photodetector probe $(0.45 \mathrm{~mm}$ in diameter) was stereotaxically placed through a skull burr hole ( $1 \mathrm{~mm}$ in diameter) in the frontoparietal cortex (1.3 $\mathrm{mm}$ posterior, $2.8 \mathrm{~mm}$ lateral to the bregma, and $1.0 \mathrm{~mm}$ below the dura). Experimental rats were then injected i.v. with recombinant human $\mathrm{SN}(50 \mu \mathrm{g}$ in $500 \mu \mathrm{l}$ saline; ProSpec-Tany TechnoGene) or vehicle (500 $\mu \mathrm{l}$ saline) 30 minutes after MCA ligation through a 26-gauge syringe into the right femoral vein. After 90 minutes of ischemia, the 10-0 suture on the MCA and arterial clips on CCAs were removed to allow for reperfusion. During recovery from anesthesia, body temperature was maintained at $37^{\circ} \mathrm{C}$ with a heat lamp.
In order to investigate the expression of $\mathrm{SN}$ after cerebral ischemia in rats, IHC analysis of rat ischemic brain and quantitative measurement of SN level in brain samples and sera by ELISA were performed on the experimental rats, as described above.

Neurological behavioral measurements. Behavioral assessments were performed 3 days before cerebral ischemia and 72 hours after cerebral ischemia. The tests measured body asymmetry and locomotor activity as previously described (51). Furthermore, grip strength was analyzed using Grip Strength Meter (TSE-Systems) as previously described with modification (52). In brief, the percentage of improvement in grip strength was measured on each fore limb separately and was calculated as the ratio between mean strength of 20 pulls of the side contralateral to the ischemia and the ipsilateral side. In addition, the ratio of grip strength after treatment to baseline was also calculated, and changes were presented as percent of baseline. In order to demonstrate whether the neuroplastic effect of $\mathrm{SN}$ in the ischemic brain was exerted through activating the Jak2/Stat3 pathway, experimental rats in the SN-treated group were injected with Jak2 inhibitor (AG490, $5 \mathrm{mg} / \mathrm{kg}$ i.p.; Calbiochem; or TG101209, $100 \mathrm{mg} / \mathrm{kg}$ i.v.; TargeGen) as previously described $(53,54)$. The neurological behavior of these rats was then measured as described above to compare with the control group.

Triphenyltetrazolium chloride staining. Two days after cerebral ischemia, animals were intracardially perfused with saline. The brain tissue was removed, immersed in cold saline for 5 minutes, and sliced into $2.0-\mathrm{mm}$ thick sections (7 slices per rat). The brain slices were incubated in $20 \mathrm{~g} / 1$ triphenyltetrazolium chloride (Research Organics Inc.), dissolved in saline for 30 minutes at $37^{\circ} \mathrm{C}$, and then transferred to a $5 \%$ formaldehyde solution for fixation. The area of infarction in each slice was measured with a digital scanner as described previously (55). The volume of infarction was obtained from the product of average slice thickness $(2 \mathrm{~mm})$ and by examining infarcted areas in all brain slices. To minimize any artifacts induced by postischemic edema in the infarcted tissue, the area of infarction was also calculated as previously described (56). To measure the infarcted area in the right cortex, we subtracted the noninfarcted area in the right cortex from the total cortical area of the left hemisphere.

Measurement of the infarct size using MRI. MRI was performed on rats under anesthesia in a General Electric imaging system (R4; GE) at 3.0 T. Brains were scanned in 6-8 coronal image slices, each $2 \mathrm{~mm}$ thick without any gaps. T2-weighted imaging pulse sequences were obtained with the use of a spin-echo technique (repetition time, 4,000 ms; echo time, $105 \mathrm{~ms}$ ) and were captured sequentially for each animal at 1,7 , and 28 days after cerebral ischemia. To measure the infarction area in the right cortex, we subtracted the noninfarcted area in the right cortex from the total cortical area of the left hemisphere. The area of infarct was drawn manually from slice to slice, and the volume was then calculated by internal volume analysis software (Voxtool; GE).

${ }^{18}$ FDG-PET examination. To assess the metabolic activity and synaptic density of brain tissue, experimental rats were examined using microPET scanning of ${ }^{18} \mathrm{FDG}$ to measure relative metabolic activity as previously described (57). In brief, ${ }^{18} \mathrm{~F}$ was produced by the ${ }^{18} \mathrm{O}(\mathrm{p}, \mathrm{n}){ }^{18} \mathrm{~F}$ nuclear reaction in a cyclotron at Tzu-Chi General Hospital and Tzu-Chi University, and ${ }^{18} \mathrm{FDG}$ was synthesized as previously described (58) with an automated ${ }^{18}$ FDG synthesis system (Nihonkokan). Data were collected with high-resolution small-animal PET (microPET Rodent R4; Concorde Microsystems Inc.). The system parameters were described by Visnyei et al. (57). After 1 week of each treatment, animals anesthetized with chloral hydrate $(0.4 \mathrm{~g} / \mathrm{kg}$ i.p.) were fixed in a customized stereotactic head holder and positioned in the microPET scanner. The animals were then given an i.v. bolus injection of ${ }^{18} \mathrm{FDG}(200-250 \mu \mathrm{Ci} / \mathrm{rat})$ dissolved in $0.5 \mathrm{ml}$ saline. Data acquisition began simultaneously with injections 
and continued for 60 minutes in 1 bed position using a 3D acquisition protocol. The image data acquired from microPET were displayed and analyzed by IDL version 5.5 (Research Systems) and ASIPro version 3.2 (Concorde Microsystems) software. Coronal sections for striatal and cortical measurements represented brain areas between 0 and $+1 \mathrm{~mm}$ from the bregma, while those for thalamic measurements represented areas between -2 and $-3 \mathrm{~mm}$ from the bregma, as estimated by visual inspection of the unlesioned side. The relative metabolic activity in regions of interest of the striatum and cortex was expressed as percentage deficit, as previously described with modification (57).

Blood pressure, heart rate, blood glucose, and blood gas measurement. Physiological parameters were measured in 7 each of control and $\mathrm{SN}$ rats. The procedure has been described previously (59).

Activated caspase-3 immunostaining and Western blot analysis. Eight hours after ischemia, rats were anesthetized with chloral hydrate and perfused with $4 \%$ paraformaldehyde. Brain slices were incubated with primary antibody against caspase-3 (cleaved caspase-3 antibody, D175, dilution 1:500; Cell Signaling) conjugated with Cy3 (1:500; Jackson Immunoresearch) for 20 hours at $4^{\circ} \mathrm{C}$, washed 3 times with PBS, and then observed by fluorescent microscopy (Axiovert 200M; Carl Zeiss). The extent of apoptosis was measured as the number of caspase- $3^{+}$apoptotic cells per square millimeter. In addition, apoptosis-related protein expression (Bcl-2, Bcl-xL, Bax, and $\mathrm{Bad}$ ) and signal transduction protein ( $\mathrm{p}$-Stat 3 ) in the right cortex and striatum region was also examined in the $\mathrm{SN}$-treated and control rats using Western blot analysis as described previously (60).

TUNEL histochemistry. To detect cellular apoptosis, a TUNEL staining Kit (DeadEnd Fluorimetric TUNEL system; Promega) was used for the TUNEL assay. Twenty-four hours after ischemia, rat brains were fixed by transcardial perfusion with saline and immersed in $4 \%$ paraformaldehyde. After brains had been frozen on dry ice, a series of adjacent $10-\mu \mathrm{m}$-thick sections were cut in the coronal plane with a cryostat. The staining and semiquantitating procedure was performed as previously described (61).

BrdU labeling and BrdU IHC. BrdU, a thymidine analog that is incorporated into the DNA of dividing cells during S-phase, was used for mitotic labeling (Sigma-Aldrich). The labeling protocol has been described previously (62). The BrdU immunostaining procedure with a specific antibody against BrdU (1:400; Boehringer Mannheim) and quantification of BrdU-immunoreactive cells have also been described previously (62). In brief, experimental rats' brains were fixed by transcardial perfusion with saline, followed by perfusion and immersion in $4 \%$ paraformaldehyde. Subsequently, the brain samples were dehydrated in $30 \%$ sucrose. After brains had been frozen on dry ice, a series of adjacent 6 - $\mu \mathrm{m}$-thick sections were cut in the coronal plane with a cryostat, stained with H\&E, and observed by light microscopy (E600; Nikon). For BrdU immunostaining, DNA was denatured by incubating each section in $50 \%$ formamide in $2 \times$ standard saline citrate at $65^{\circ} \mathrm{C}$ for 2 hours and then in $2 \mathrm{~N} \mathrm{HCl}$ at $37^{\circ} \mathrm{C}$ for 30 minutes, then rinsed in $0.1 \mathrm{M}$ boric acid with $\mathrm{pH}$ 8.5. Sections were rinsed with Tris buffer and treated with $1 \%$ $\mathrm{H}_{2} \mathrm{O}_{2}$ to block endogenous peroxidase. The immunostaining procedure was performed using the labeled streptavidin-biotin method (DAKO LSAB-2 Kit, Peroxidase; DAKO). Tissue slides were incubated with the appropriate diluted antibodies to BrdU (for nuclear identification, dilution 1:400; Boehringer Mannheim) at room temperature for 1 hour. After washing with Tris-buffered saline containing $0.1 \%$ Tween- 20 , the specimens were sequentially incubated for 10-30 minutes with biotinylated anti-rabbit and anti-mouse (1:200; R\&D Systems) immunoglobulins and peroxidase-labeled streptavidin. Next, a pulse labeling method $(n=16)$ was used to observe the time course of cell proliferation in the brain after cerebral ischemia. Experimental rats were injected with BrdU $(50 \mathrm{mg} / \mathrm{kg}$ i.p.) every 4 hours for 12 hours before sacrifice. A cumulative labeling method $(n=16)$ was used to examine the population of proliferative cells during 14 days of cerebral ischemia. Rats received daily injections of $\mathrm{BrdU}(50 \mathrm{mg} / \mathrm{kg}$ i.p.) for 14 consecutive days, starting the day after MCA ligation, and were sacrificed 14 days after the last injection.

Transgenic GFP-chimeric mouse preparation. In order to verify the enhancement of the INPC and BMSC mobilization and homing into brain by $\mathrm{SN}$ administration, a bone marrow sample was removed from the long bones of adult male donor mice as previously reported (63). Both ends of the femur and tibia were penetrated using a syringe with a 25 -gauge needle, and the marrow was flushed out with sterile saline. Total marrow from 1 femur was diluted to $1 \mathrm{ml}$ and then strained through $30-\mu \mathrm{m}$ Spectramesh (Fisher Scientific). Before bone marrow transplantation, female recipient mice underwent whole-body gamma irradiation with ${ }^{137} \mathrm{Cs}$ using a Gammacell 40 irradiator (MDS Nordion). A total dose of 9 Gy was administered to ablate the whole bone marrow. The mice received rescuing bone marrow transplantations within 24 hours of irradiation. Donor bone marrow was injected into the recipient animal's tail as an 80- $\mu 1$ cell suspension containing $3 \times 10^{6}$ cells. At 3 weeks after transplantation, mice were anesthetized with chloral hydrate $(0.3 \mathrm{~g} / \mathrm{kg}$ i.p. $)$ and subjected to right MCA ligation and bilateral CCA clamping for $60 \mathrm{~min}$ utes, as previously described with modification (50). At 60 minutes after arterial ligation, experimental mice were injected i.v. with recombinant human SN (20 $\mu \mathrm{g}$ in $200 \mu \mathrm{l}$ saline; Neosystems) or vehicle (200 $\mu \mathrm{l}$ saline) through a 30-gauge syringe into the femoral vein. BrdU labeling was also performed for each mouse as described above.

Laser-scanning confocal microscopy for double-immunofluorescence analysis. To identify the coexpression of cell type-specific markers in SN-immunoreactive $\mathrm{GFP}^{+}$and $\mathrm{BrdU}^{+}$cells, immunofluorescent colocalization study with $3 \mathrm{D}$ images was performed to test for the expression of GFAP, $\alpha$-SMA, vWF, MAP-2, Musashi-1, and Neu-N. The double-immunofluorescence technique with specific antibodies against BrdU (1:400; Mannheim), GFAP (1:400; Sigma-Aldrich), MAP-2 (1:200, Boehringer Mannheim), Nestin (1:400, Sigma-Aldrich), Neu-N (1:200, Chemicon), vWF (1:400, SigmaAldrich), Musashi-1 (1:100, Serotec) and SMA (1:100, BD Pharmingen) conjugated with Cy3, Cy5, or FITC (1:500, Jackson Immunoresearch) has been described previously (14). The tissue sections were analyzed with a Carl Zeiss LSM510 laser-scanning confocal microscope.

Angiogenic evaluation by FITC-dextran perfusion and CD31 IHC. In order to examine the blood vessels, cerebral microcirculation was analyzed by administering a fluorescent plasma marker (FITC-dextran; Sigma-Aldrich) to rats i.v. and observing them under fluorescent microscopy (Axiovert 200M; Carl Zeiss), as previously described (64). In addition, to quantify the cerebral blood vessel density and examine vascular remodeling by macrophage, experimental rats were anesthetized with chloral hydrate and perfused with $4 \%$ paraformaldehyde. Histological sections $(6 \mu \mathrm{m})$ were stained with specific antibody to CD-31 (1:100; BD Biosciences - Pharmingen) conjugated with Cy-3 (1:500; Jackson Immunoresearch). The number of blood vessels was determined as previously described (30).

Measurement of CBF. Experimental rats were positioned in a stereotaxic frame, and baseline local cortical blood flow was monitored after cerebral ischemia with a laser Doppler flowmeter (LDF monitor; Moor Instruments) in anesthetized state (chloral hydrate) as previously described (65). In brief, $\mathrm{CBF}$ values were calculated as percentage increase over baseline local cortical blood flow.

Statistics. In this study, all observers were blind to the actual treatment result measurement. Sample sizes in animal treatment groups were monitored to ensure sufficient discriminatory power with Minitab, version 15, and were calculated to provide $80 \%$ power, sufficient to detect differences between each treatment. Results are expressed as mean \pm SEM of all measurements in a treatment group. The behavioral scores have been evaluated 
for normality. Two-tailed Student's $t$ tests were used to evaluate significance of mean differences between the control and the treated group. Data lacking normal distribution were analyzed by nonparametric ANOVA. $P$ values less than 0.05 were taken as significant.

\section{Acknowledgments}

We thank H. Wilson and M. Loney of Academia Sinica for their critical reading of this manuscript. This work was supported in part by research grants from the Chen-Han Foundation for Education, Academia Sinica (94M003), and the National Science Council (NSC95-2314-B-303-003).
Received for publication May 18, 2007, and accepted in revised form October 3, 2007.

Address correspondence to: Hung Li, Institute of Molecular Biology, Academia Sinica, 128 Sec. 2, Academia Road, Nankang, Taipei 11529, Republic of China. Phone: 886-2-2788-0460; Fax: 886-2-2782-6085; E-mail: hungli@ccvax.sinica.edu.tw. Or to: Woei-Cherng Shyu, Center for Neuropsychiatry, China Medical University and Hospital, 2 Yuh-Der Road, Taichung, Taiwan 40447, Republic of China. Phone: 886-4-2205-2121 ext. 6034; Fax: 886-4-2208-0666; E-mail: shyu9423@yahoo.com.tw.
1. Fischer-Colbrie, R., Laslop, A., and Kirchmair, R. 1995. Secretogranin II: molecular properties, regulation of biosynthesis and processing to the neuropeptide secretoneurin. Prog. Neurobiol. 46:49-70.

2. Cozzi, M.G., et al. 1989. Immunohistochemical localization of secretogranin II in the rat cerebellum. Neuroscience. 28:423-441.

3. Lechner, T., et al. 2004. Chromogranin peptides in Alzheimer's disease. Exp. Gerontol. 39:101-113.

4. Marksteiner, J., Kaufmann, W.A., Gurka, P., and Humpel, C. 2002. Synaptic proteins in Alzheimer's disease. J. Mol. Neurosci. 18:53-63.

5. Kaufmann, W.A., et al. 1998. Synaptic loss reflected by secretoneurin-like immunoreactivity in the human hippocampus in Alzheimer's disease. Eur. J. Neurosci. 10:1084-1094.

6. Gasser, M.C., et al. 2003. Secretoneurin promotes pertussis toxin-sensitive neurite outgrowth in cerebellar granule cells. J. Neurochem. 85:662-669.

7. Marti, E., Ferrer, I., and Blasi, J. 2001. Differential regulation of chromogranin $A$, chromogranin $B$ and secretoneurin protein expression after transient forebrain ischemia in the gerbil. Acta. Neuropathol. 101:159-166.

8. Nishimura, M., et al. 1994. Synaptophysin and chromogranin A immunoreactivities of Lewy bodies in Parkinson's disease brains. Brain Res. 634:339-344.

9. Marti, E., Blasi, J., and Ferrer, I. 2002. Early induction of secretoneurin expression following kainic acid administration at convulsant doses in the rat and gerbil hippocampus. Hippocampus. 12:174-185.

10. Pirker, S., et al. 2001. Chromogranins as markers of altered hippocampal circuitry in temporal lobe epilepsy. Ann. Neurol. 50:216-226.

11. van Gijn, J., and Dennis, M.S. 1998. Issues and answers in stroke care. Lancet. 352(Suppl. 3):SIII23-SIII27.

12. Bang, O.Y., Lee, J.S., Lee, P.H., and Lee, G. 2005. Autologous mesenchymal stem cell transplantation in stroke patients. Ann. Neurol. 57:874-882.

13. Chen, J., et al. 2001. Therapeutic benefit of intravenous administration of bone marrow stromal cells after cerebral ischemia in rats. Stroke. 32:1005-1011.

14. Li, Y., et al. 2002. Human marrow stromal cell therapy for stroke in rat: Neurotrophins and functional recovery. Neurology. 59:514-523.

15. Walter, D.H., et al. 2002. Statin therapy accelerates reendothelialization: a novel effect involving mobilization and incorporation of bone marrowderived endothelial progenitor cells. Circulation. 105:3017-3024.

16. Werner, N., et al. 2003. Intravenous transfusion of endothelial progenitor cells reduces neointima formation after vascular injury. Circ. Res. 93:e17-e24.

17. Shyu, W.C., et al. 2004. Functional recovery of stroke rats induced by granulocyte colony-stimulating factor-stimulated stem cells. Circulation. 110:1847-1854.

18. Kirchmair, R., et al. 2004. Secretoneurin, an angiogenic neuropeptide, induces postnatal vasculogenesis. Circulation. 110:1121-1127.
19. Kahler, C.M., et al. 1997. Inhibition of proliferation and stimulation of migration of endothelial cells by secretoneurin in vitro. Arterioscler. Thromb. Vasc. Biol. 17:932-939.

20. Jung, K.H., et al. 2006. Granulocyte colony-stimulating factor stimulates neurogenesis via vascular endothelial growth factor with STAT activation. Brain Res. 1073-1074:190-201.

21. Wang, M., et al. 2007. STAT3 mediates bone marrow mesenchymal stem cell VEGF production. J. Mol. Cell. Cardiol. 42:1009-1015.

22. Jogi, A., et al. 2004. Human neuroblastoma cells exposed to hypoxia: induction of genes associated with growth, survival, and aggressive behavior. Exp. Cell Res. 295:469-487.

23. Klein, S.M., et al. 2005. GDNF delivery using human neural progenitor cells in a rat model of ALS. Hum. Gene Ther. 16:509-521.

24. Srinivasan, S., Anitha, M., Mwangi, S., and Heuckeroth, R.O. 2005. Enteric neuroblasts require the phosphatidylinositol 3-kinase/Akt/Forkhead pathway for GDNF-stimulated survival. Mol. Cell. Neurosci. 29:107-119.

25. Kirchmair, R., et al. 2004. The neuropeptide secretoneurin acts as a direct angiogenic cytokine in vitro and in vivo. Circulation. 109:777-783.

26. Lu, P., Jones, L.L., Snyder, E.Y., and Tuszynski, M.H. 2003. Neural stem cells constitutively secrete neurotrophic factors and promote extensive host axonal growth after spinal cord injury. Exp. Neurol. 181:115-129.

27. Humpel, C., et al. 1994. Neurons of the hippocampal formation express glial cell line-derived neurotrophic factor messenger RNA in response to kainate-induced excitation. Neuroscience. 59:791-795.

28. Knuckey, N.W., et al. 1996. Differential neuronal and astrocytic expression of transforming growth factor beta isoforms in rat hippocampus following transient forebrain ischemia. Brain Res. Mol. Brain Res. 40:1-14.

29. Endres, M., et al. 1998. Attenuation of delayed neuronal death after mild focal ischemia in mice by inhibition of the caspase family. J. Cereb. Blood Flow Metab. 18:238-247.

30. Taguchi, A., et al. 2004. Administration of CD34+ cells after stroke enhances neurogenesis via angiogenesis in a mouse model. J. Clin. Invest. 114:330-338.

31. Emanueli, C., et al. 2002. Nerve growth factor promotes angiogenesis and arteriogenesis in ischemic hindlimbs. Circulation. 106:2257-2262.

32. Zukowska-Grojec, Z., et al. 1998. Neuropeptide $Y$ : a novel angiogenic factor from the sympathetic nerves and endothelium. Circ. Res. 83:187-195.

33. Movafagh, S., Hobson, J.P., Spiegel, S., Kleinman, H.K., and Zukowska, Z. 2006. Neuropeptide Y induces migration, proliferation, and tube formation of endothelial cells bimodally via Y1, Y2, and Y5 receptors. FASEB J. 20:1924-1926.

34. Sondell, M., Lundborg, G., and Kanje, M. 1999. Vascular endothelial growth factor has neurotrophic activity and stimulates axonal outgrowth, enhancing cell survival and Schwann cell prolifera- tion in the peripheral nervous system. J. Neurosci. 19:5731-5740.

35. Schratzberger, P., et al. 2000. Favorable effect of VEGF gene transfer on ischemic peripheral neuropathy. Nat. Med. 6:405-413.

36. Schratzberger, P., et al. 2001. Reversal of experimental diabetic neuropathy by VEGF gene transfer. J. Clin. Invest. 107:1083-1092.

37. Asahara, T., et al. 1999. VEGF contributes to postnatal neovascularization by mobilizing bone marrow-derived endothelial progenitor cells. EMBO J. 18:3964-3972.

38. Perez, L.E., et al. 2004. Increased plasma levels of stromal-derived factor-1 (SDF-1/CXCL12) enhance human thrombopoiesis and mobilize human colony-forming cells (CFC) in NOD/SCID mice. Exp. Hematol. 32:300-307.

39. Llevadot, J., et al. 2001. HMG-CoA reductase inhibitor mobilizes bone marrow-derived endothelial progenitor cells. J. Clin. Invest. 108:399-405.

40. Heeschen, C., et al. 2003. Erythropoietin is a potent physiologic stimulus for endothelial progenitor cell mobilization. Blood. 102:1340-1346.

41. Kimura, T., et al. 2004. The sphingosine 1-phosphate receptor agonist FTY720 supports CXCR4-dependent migration and bone marrow homing of human CD34+ progenitor cells. Blood. 103:4478-4486.

42. Waskow, C., Terszowski, G., Costa, C., Gassmann, M., and Rodewald, H.R. 2004. Rescue of lethal c-KitW/ W mice by erythropoietin. Blood. 104:1688-1695.

43. Kucia, M., Ratajczak, J., Reca, R., Janowska-Wieczorek, A., and Ratajczak, M.Z. 2004. Tissue-specific muscle, neural and liver stem/progenitor cells reside in the bone marrow, respond to an SDF-1 gradient and are mobilized into peripheral blood during stress and tissue injury. Blood Cells Mol. Dis. 32:52-57.

44. Lindsberg, P.J., Carpen, O., Paetau, A., KarjalainenLindsberg, M.L., and Kaste, M. 1996. Endothelial ICAM-1 expression associated with inflammatory cell response in human ischemic stroke. Circulation. 94:939-945.

45. Goldberg, M.P., and Choi, D.W. 1993. Combined oxygen and glucose deprivation in cortical cell culture: calcium-dependent and calcium-independent mechanisms of neuronal injury. J. Neurosi. 13:3510-3524.

46. Koh, J.Y., and Choi, D.W. 1987. Quantitative determination of glutamate mediated cortical neuronal injury in cell culture by lactate dehydrogenase efflux assay. J. Neurosci. Methods. 20:83-90.

47. Wang, Y., et al. 2001. Bone morphogenetic protein6 reduces ischemia-induced brain damage in rats. Stroke. 32:2170-2178.

48. Niquet, J., Baldwin, R.A., Allen, S.G., Fujikawa, D.G., and Wasterlain, C.G. 2003. Hypoxic neuronal necrosis: protein synthesis-independent activation of a cell death program. Proc. Natl. Acad. Sci. U. S. A. 100:2825-2830.

49. Wang, Y., et al. 2007. Adaptive secretion of granulocyte-macrophage colony-stimulating factor (GMCSF) mediates imatinib and nilotinib resistance in $\mathrm{BCR} / \mathrm{ABL}+$ progenitors via JAK-2/STAT-5 pathway 
activation. Blood. 109:2147-2155.

50. Chen, S.T., Hsu, C.Y., Hogan, E.L., Maricq, H., and Balentine, J.D. 1986. A model of focal ischemic stroke in the rat: reproducible extensive cortical infarction. Stroke. 17:738-743.

51. Chang, C.F., et al. 2003. Intravenous administration of bone morphogenetic protein-7 after ischemia improves motor function in stroke rats. Stroke. 34:558-564.

52. Dunnett, S.B., Torres, E.M., and Annett, L.E. 1998 A lateralised grip strength test to evaluate unilateral nigrostriatal lesions in rats. Neurosci. Lett. 246:1-4.

53. El-Adawi, H., et al. 2003. The functional role of the JAK-STAT pathway in post-infarction remodeling. Cardiovasc. Res. 57:129-138.

54. Pardanani, A., et al. 2007. TG101209, a small molecule JAK2-selective kinase inhibitor potently inhibits myeloproliferative disorder-associated JAK2V617F and MPLW515L/K mutations. Leukemia. 21:1658-1668.

55. Wang, Y., Lin, S.Z., Chiou, A.L., Williams, L.R., and Hoffer, B.J. 1997. Glial cell line-derived neurotroph- ic factor protects against ischemia-induced injury in the cerebral cortex. J. Neurosci. 17:4341-4348.

56. Lin, T.N., He, Y.Y., Wu, G., Khan, M., and Hsu, C.Y. 1993. Effect of brain edema on infarct volume in a focal cerebral ischemia model in rats. Stroke. 24:117-121.

57. Visnyei, K., et al. 2006. Neural progenitor implantation restores metabolic deficits in the brain following striatal quinolinic acid lesion. Exp. Neurol. 197:465-474.

58. Hamacher, K., Coenen, H.H., and Stocklin, G. 1986 Efficient stereospecific synthesis of no-carrieradded 2-[18F]-fluoro-2-deoxy-D-glucose using aminopolyether supported nucleophilic substitution. J. Nucl. Med. 27:235-238.

59. Lin, S.Z., Hoffer, B.J., Kaplan, P., and Wang, Y. 1999. Osteogenic protein-1 protects against cerebral infarction induced by MCA ligation in adult rats. Stroke. 30:126-133.

60. Harada, M., et al. 2005. G-CSF prevents cardiac remodeling after myocardial infarction by activating the Jak-Stat pathway in cardiomyocytes. Nat. Med. 11:305-311.
61. Li, Y., Lu, Z., Keogh, C.L., Yu, S.P., and Wei, L. 2007. Erythropoietin-induced neurovascular protection, angiogenesis, and cerebral blood flow restoration after focal ischemia in mice. J. Cereb. Blood Flow Metab. 27:1043-1054.

62. Zhang, R.L., Zhang, Z.G., Zhang, L., and Chopp, M. 2001. Proliferation and differentiation of progenitor cells in the cortex and the subventricular zone in the adult rat after focal cerebral ischemia. Neuroscience. 105:33-41.

63. Hess, D.C., et al. 2002. Bone marrow as a source of endothelial cells and NeuN-expressing cells after stroke. Stroke. 33:1362-1368.

64. Morris, D.C., Zhang, Z., Davies, K., Fenstermacher, J., and Chopp, M. 1999. High resolution quantitation of microvascular plasma perfusion in nonischemic and ischemic rat brain by laser-scanning confocal microscopy. Brain Res. Brain Res. Protoc. 4:185-191.

65. Park, L., et al. 2005. NADPH-oxidase-derived reactive oxygen species mediate the cerebrovascular dysfunction induced by the amyloid beta peptide. J. Neurosci. 25:1769-1777. 